\title{
Understanding the role of nanostructuring in photoelectrode performance for light- driven water splitting
}

\author{
Laurence M. Peter ${ }^{1}$, Gurudayal ${ }^{2,3}$, Lydia Helena Wong $^{2}$, Fatwa F. Abdi ${ }^{4}$ \\ ${ }^{1}$ Department of Chemistry, University of Bath, Bath BA2 7AY, United Kingdom \\ ${ }^{2}$ School of Materials Science and Engineering, Nanyang Technological University, Nanyang Avenue, \\ Singapore 639798 \\ ${ }^{3}$ Joint Center for Artificial Photosynthesis, Lawrence Berkeley National Laboratory, 1 Cyclotron Road, \\ Berkeley, CA 94720, USA \\ ${ }^{4}$ Institute for Solar Fuels, Helmholtz-Zentrum Berlin für Materialien und Energie GmbH, Hahn-Meitner-Platz \\ 1, Berlin 14109, Germany
}

\begin{abstract}
The analysis of capacitance data for regular nanostructured photoelectrodes is revisited using a hematite nanorod array as an example. The effects of the cylindrical nanorod geometry on the capacitance-voltage behaviour are outlined, and the limiting case of complete depletion is discussed in terms of the residual geometric capacity at the base of the nanorods. Since nanorod arrays generally leave areas of the substrate exposed, it is necessary to consider the parallel capacitance associated with the fraction of uncovered surface. The sensitivity of the capacitance fitting to parameter variation is explored. The enhancement of external quantum efficiency (EQE) by nanostructuring is also discussed using hematite nanorod arrays as experimental examples. It is shown that, although very substantial EQE enhancement should be achieved by simple geometric effects, the performance of nanostructured hematite electrodes in the visible region of the spectrum is considerably lower than predicted if all charge carriers generated in the space charge region (SCR) were collected. Further analysis reveals that the internal quantum efficiency increases with photon energy, suggesting that the probability of generating free, rather than bound, electron-hole pairs in hematite depends on the excess energy hv - $E_{\text {gap }}$.
\end{abstract}

\section{Introduction}

Against a background of increasing deployment of photovoltaics for electricity generation, light-driven water splitting to generate hydrogen as a fuel and feedstock remains an important goal of energy research since it addresses the key problem of energy storage. The search for stable photoelectrodes with suitable optoelectronic and chemical properties has occupied electrochemists for decades. Since light-driven water splitting reactions involve minority carriers (holes for oxidation at n-type photoanodes and electrons for reduction at p-type photocathodes), we would like ideally to use semiconductors that have long minority carrier lifetimes, high carrier mobilities and suitable light harvesting properties. Unfortunately, while the best materials from an optoelectronic point of view (for example, silicon, III-V and II-VI semiconductors and, more recently, the lead halide perovskites) can be used to fabricate efficient solar cells, as photoelectrodes, they are prone to photo-corrosion and must therefore be protected by a stable impervious layer such as $\mathrm{TiO}_{2}$. This configuration corresponds to a 'buried' junction in which the generated photovoltage is used to drive electrolysis at metallic or pseudo-metallic electrodes.[1,2] By contrast, photoelectrolysis systems in which the light harvesting semiconductor is in direct contact with the electrolyte are more demanding in terms of stability as well as band alignment with the oxygen and hydrogen redox Fermi 
levels. For this reason, oxide materials such as hematite $\left(\alpha-\mathrm{Fe}_{2} \mathrm{O}_{3}\right)[3-6]$ and bismuth vanadate $\left(\mathrm{BiVO}_{4}\right)$ [7-9] have been studied widely, despite their rather poor optoelectronic properties (hematite, for example, is an indirect n-type bandgap semiconductor with a very short hole lifetime and low hole mobility).

An often-cited figure of merit in this context is the minority carrier diffusion length, which in the neutral bulk of a semiconductor is defined as $L_{\min }=\left(D_{\min } \tau_{\min }\right)^{1 / 2}$, where $\tau_{\min }$ is the minority carrier lifetime and $D_{\min }$ is the minority carrier diffusion coefficient, which is related to the mobility $\mu_{\min }$ by $D_{\min }=\left(k_{B} T / q\right) \mu_{\min }$. Based on early studies, hole diffusion lengths for hematite are believed to be in the range of a few $\mathrm{nm},[10,11]$ many orders of magnitude smaller than minority carrier diffusion lengths in III-V semiconductors such as GaAs, for example, which can be several microns.[12] A low hole diffusion length means that the separation and collection of photogenerated electron-hole pairs that is essential for any photovoltaic of photoelectrochemical device to function only takes place in regions that are strongly depleted of majority carriers. In the case of a conventional planar hematite photoelectrode, this means that only photons absorbed in the space charge region (SCR) of the semiconductor/electrolyte contact contribute to the photocurrent. Nearly all holes generated in the quasi-neutral region (QNR) recombine very rapidly with electrons, which are present in large excess. Only holes generated very close (i.e. within one ca. diffusion length) to the edge of the SCR have any chance of being collected at the semiconductor/electrolyte interface. Since the penetration depth of the incident illumination (typically close to $1 \mu \mathrm{m}$ ) is generally much greater than the width of the space SCR (a few tens of nm for a typical hematite electrode), the external quantum efficiency (EQE) of the photocurrent is much less than $100 \%$. However, as we shall see below, even the assumption that all carriers generated in the SCR are collected is open to doubt in the case of hematite.

A popular approach aimed at tackling this problem of mismatched characteristic lengths is to nanostructure the water-splitting photoelectrode. The often-repeated argument is that if the scale of the nanostructure is comparable with the minority carrier diffusion length, this will enhance the chance of photogenerated minority carriers reaching the semiconductor/electrolyte interface, increasing the EQE. We show here that this interpretation of the beneficial effects of nanostructuring does not stand up to scrutiny. For highly doped materials like hematite (here we take highly doped to mean a doping density greater than $10^{18} \mathrm{~cm}^{-3}$ ), we show that it is the collection of holes in the SCR and not the hole diffusion length in QNR that explains why nanostructuring can enhance the EQE.

To be able to model the EQE of regular nanostructured systems such as nanorod arrays with cylindrical geometry, we need to have reliable estimates of the doping density. Reliable determination of the doping density, $N_{d}$, is important since it allows us to distinguish between cases where the space charge layer thickness is either smaller than or larger than the characteristic lengths of the nanostructures. However, the conventional Mott Schottky analysis used to determine $N_{d}$ for planar electrodes cannot be applied to non-planar electrodes. Instead, the geometry (spherical, cylindrical etc.) of the nanostructure needs to be considered. In addition, we need to have some idea of how rapidly minority carriers are consumed at the interface with the electrolyte, either by reaction with electrolyte species or by recombination with majority carriers. The well-known analysis of the external 
photocurrent quantum efficiency by Gärtner[13] assumes that the interface acts as a perfect sink for minority carriers. However, water splitting involves multiple electron/proton transfer steps, and the reaction of holes in the photooxidation of water, for example, is remarkably slow. It follows that the rate of the water splitting reaction cannot be controlled by the rate of hole transport to the interface (except as insofar as this affects recombination in the SCR: see below). Consequently, arguments about the benefits of nanostructuring that are based on matching the feature sizes of the electrode to the diffusion length of holes in the QNR are unconvincing. In this paper, we explore some of the difficulties encountered in the determination of doping densities for a hematite nanorod array and discuss an alternative explanation for the superior performance of nanostructured oxide electrodes. We also examine the performance of hematite nanorod electrodes by comparing it with the performance predicted by assuming that all electron-hole pairs generated in the SCR are collected. We show that recombination in the SCR is a likely to be a major limiting factor in the performance of hematite electrodes.

\section{Capacitance measurements of nanostructured semiconductor electrodes}

\subsection{The planar case}

We begin by revisiting the classical theory of the semiconductor electrolyte interface before moving on to discuss the case of an array of cylindrical nanorods. For simplicity, we initially ignore the effects of inversion, which are discussed later. Instead we assume that deep depletion is possible because the rate of thermal generation of minority carriers is smaller than the rate of their removal at the electrolyte contact. Furthermore, we omit consideration of surface states and Fermi level pinning.

The width of the SCR, $W_{S C R}$, formed at a planar semiconductor-electrode junction depends on the doping density $\left(N_{d}\right)$, the relative permittivity $(\varepsilon)$ of the material and the potential drop, $\Delta \phi_{S C R}$, across the space charge region.

$$
W_{S C R}=\left(\frac{2 \Delta \phi_{S C R} \varepsilon \varepsilon_{0}}{q N_{d}}\right)^{1 / 2}
$$

For low-doped semiconductors, this potential drop is equal to the difference between the applied potential $E$ and the flat band potential $E_{f b}$, but in the cases that we consider here, the doping is sufficiently high that a significant part of the change in potential difference with applied voltage appears across the Helmholtz double layer. Following the approach of De Gryse et al.,[14] we therefore separate the overall potential drop into two terms.

$$
E-E_{f b}=\Delta \phi_{S C R}+\Delta \phi_{H}
$$

Treating the Helmholtz capacitance $C_{H}$ as constant (capacitance and charge are expressed per unit area), the potential drop, $\Delta \phi_{H}$, across the Helmholtz layer is given by

$$
\Delta \phi_{H}=\frac{Q_{S C R}}{C_{H}}
$$

where $Q_{S C R}$, the charge in the SCR, is given in the Mott Schottky approximation by

$$
Q_{S C R}=\left(2 q \varepsilon \varepsilon_{0} N_{d}\right)^{1 / 2}\left(\Delta \phi_{S C R}-\frac{k_{B} T}{q}\right)^{1 / 2}
$$


The potential drop across the Helmholtz layer therefore changes according to the expression[14]

$$
\Delta \phi_{H}=\left(\frac{2 q \varepsilon \varepsilon_{0} N_{d}}{C_{H}^{2}}\right)\left(\Delta \phi_{S C R}-\frac{k_{B} T}{2}\right)^{1 / 2}
$$

From equation 2 and equation 5 we obtain a more general form of the Mott Schottky equation that is applicable to highly-doped electrodes in the deep depletion approximation (i.e. ignoring the effects of inversion).[14]. Equation 5 must be replaced by the more general form

$$
\frac{1}{C^{2}}=\frac{1}{C_{H}^{2}}+\frac{2}{q \varepsilon \varepsilon_{0} N_{d}}\left(E-E_{f b}-\frac{k_{B} T}{q}\right)
$$

where $C$ is the measured total capacitance.[14] It can be seen from equation 6 that the Mott Schottky plot passes through the point $1 / C_{H}{ }^{2}$ on the $y$-axis when $E-E_{f b}-k_{B} T / q=0$ and intercepts the voltage axis at an electrode potential $E_{0}$ given by

$$
E_{0}=E_{f b}+\frac{k_{B} T}{q}-\frac{q \varepsilon \varepsilon_{0 N_{d}}}{2 C_{H}^{2}}
$$

Equation 6 was derived for deep depletion conditions. However, for the case of hematite under water splitting conditions, the deep depletion condition cannot be achieved, as demonstrated by the fact that water oxidation occurs in the dark. This process must involve holes generated at the surface by thermal excitation of electrons from the valence band or by tunnelling of electrons through a narrow space charge region from the valence band at the surface. The latter process could be assisted by thermal excitation of electrons to surface states located in the band gap. The presence of mobile holes gives rise to a very narrow charge region and a correspondingly high capacitance, as shown below in Figure 1. Under these conditions most of the potential drop will occur in the Helmholtz layer, and the electrode will behave more like a metal.

The general expressions for the space charge capacitance including accumulation depletion and inversion conditions can be found in textbooks, for example Sato.[15] Figure 1 illustrates the three regions in the capacitance plot calculated for the case of n-type hematite with a doping density of $10^{19} \mathrm{~cm}^{-3}$ assuming thermal equilibrium is established. In what follows, we restrict attention to the depletion region, but note that this region will be limited by the onset of inversion when the band bending exceeds half the band gap. 


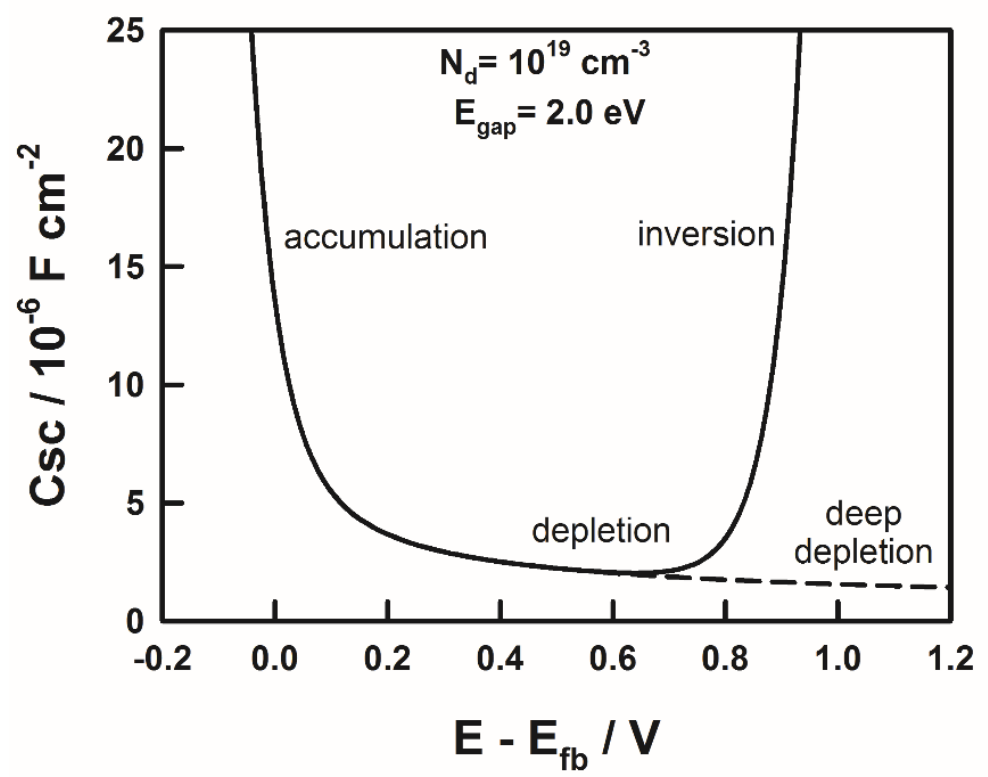

Figure1. Capacitance voltage plot calculated for hematite to illustrate the accumulation, depletion and inversion regions. Donor density as shown.

\subsection{The cylindrical case}

An example of the kind of nanorod array electrode that we have chosen to look at in detail in this paper is shown in Figure 2. It consists of a regular array of approximately cylindrical hematite nanorods with an average diameter of around $50 \mathrm{~nm}$ and a length of ca. $350 \mathrm{~nm}$ grown on an FTO substrate by a hydrothermal process followed by annealing at $550^{\circ} \mathrm{C} .[16]$
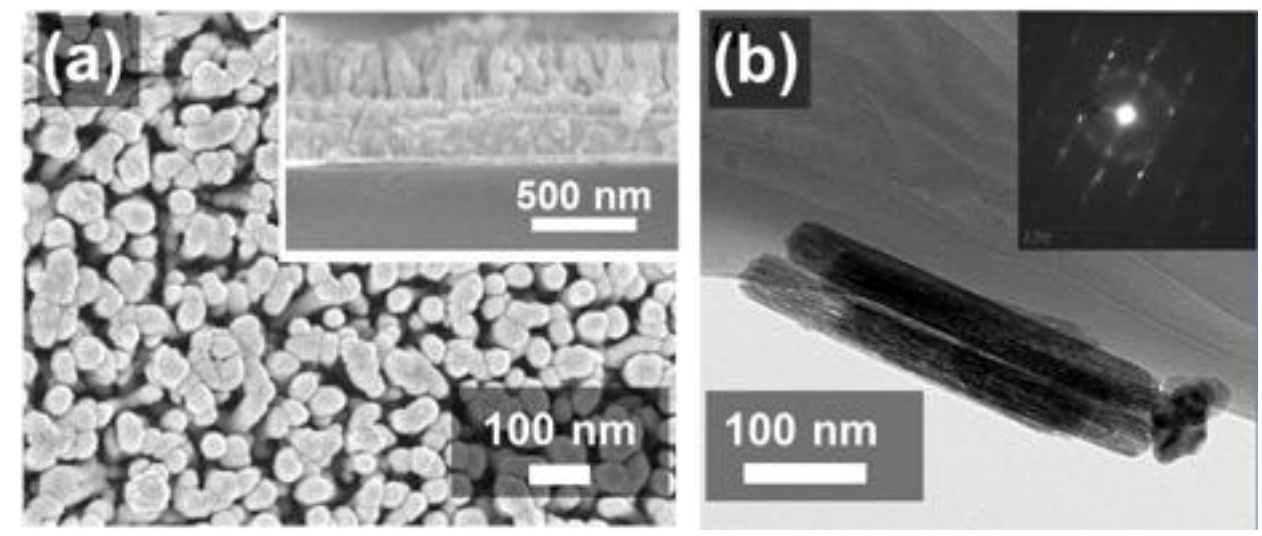

Figure 2. Hematite nanorod array grown on an FTO substrate. (a) Plane view image of the electrode showing the nanorod packing. The inset shows the cross-sectional FESEM image of the sample. (b)TEM image of a single nanorod and the respective selective area electron diffraction pattern showing the degree of crystallinity.

These nanorod electrodes have been characterized by measuring their capacitance as a function of electrode potential,[17] and a typical result is shown in the Mott Schottky plot in Figure 3. The plot appears to have a linear section, although it shows clear evidence for inversion at potentials beyond ca. $1.2 \mathrm{~V}$ vs. RHE since the fall in $1 / \mathrm{C}^{2}$ corresponds to the rapid rise in electrode capacitance under inversion conditions that is shown in Figure 1. It would be tempting to ignore the cylindrical geometry and fit the data to equation 6 for a planar electrode as shown by the broken line. As we show below, this approach implicitly assumes that the space charge layer thickness is much smaller than the rod radius over the voltage range where the linear fit is made. If we take this simple approach, we need to 
consider the true surface area of the electrode when calculating the doping density. For high aspect ratio nanorods, the ratio of the internal surface area to the geometric area is given to a good approximation by $A=2 \pi N_{\text {rod }} R L$, where $N_{\text {rod }}$ is the number density of rods of radius $R$ and length $L$. For values estimated for the array shown in Figure $2\left(N_{\text {rod }}=3.5 \times 10^{10} \mathrm{~cm}^{-2}, R\right.$ $=25 \mathrm{~nm}, L=350 \mathrm{~nm}$ ), the ratio is 19.2 . If we take this ratio and a reasonable value of 33 for the relative permittivity of hematite,[18] the doping density obtained from equation 6 is $6 \times$ $10^{18} \mathrm{~cm}^{-3}$. At first sight, this value seems reasonable, but as a quick check for consistency we could estimate the width of the space charge region using equation 1 . The apparent flat band potential from the linear fit in Figure 3 is $0.5 \mathrm{~V}$ vs RHE, so a potential of $1.25 \mathrm{~V}$ (the end of the linear fit) corresponds to a band bending of $0.75 \mathrm{eV}$. The width of the space charge region predicted by equation 1 is then $24 \mathrm{~nm}$, which is almost equal to the mean radius of the rods. The test for consistency has apparently been failed since the condition $W_{S C R}<R$ is not met. However, as we show below, this test can be misleading since the width of the space charge region in a cylindrical nanorod is not described by equation 1 .

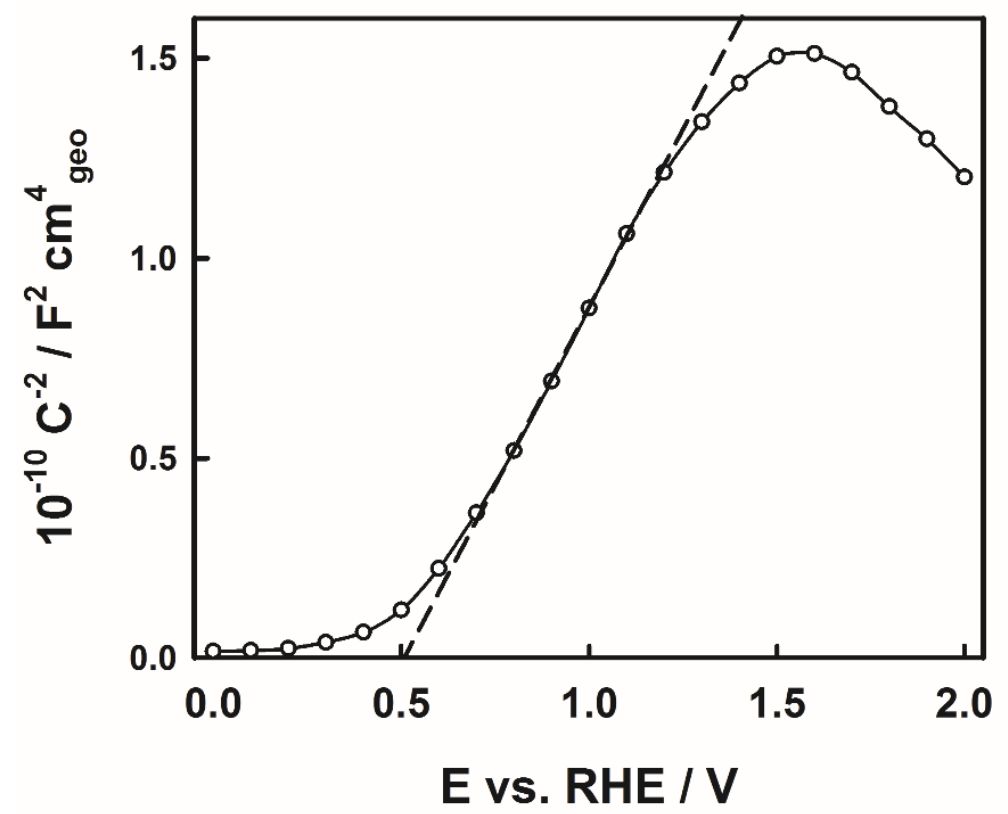

Figure 3. Experimental Mott Schottky plot for the undoped hematite nanorod array electrode illustrated in Figure 2. Electrolyte 1.0 M NaOH. Note that the capacitance values are expressed in terms of the geometric area of the electrode rather than the true internal surface area, which is around 20 times larger. For calculations of doping based on the conventional Mott Schottky equation (i.e. assuming that the width of the space charge region is much smaller than the rod radius), it is necessary to take the internal surface area into account.

Having shown that the planar approximation breaks down, we move on to consider the cylindrical geometry. We consider high aspect ratio nanorods and ignore the contribution from the tips. The potential distribution and space charge capacitance of a single nanorod have been discussed in terms of an analytical model by Mora-Sero et al.[19] and by TenaZaera et al.[20] in studies of electrodeposited $\mathrm{ZnO}$ nanorod arrays. Tornow et al.[21] have also analysed the capacitance behaviour of $\mathrm{ZnO}$ nanorods prepared by chemical bath deposition and have compared the results of analytical and more exact numerical finite element modelling. Although the work of Mora-Sero et al. is widely cited in papers dealing with nanorod arrays for water splitting, few attempts have been made to apply the modelling approach quantitatively. Here we explore the approach developed by these authors and extend it to model the EQE response of nanorod arrays. We find that the expressions given in 
Mora-Sero et al.[19] for the voltage drop across the space charge layer and for the space charge capacitance are incorrect. The correct expressions and their derivation are given in the following section.

We begin by applying the abrupt depletion approximation (the limits of this approximation when applied to the cylindrical case have been discussed by Tornow et al.[21]). As shown in Figure 4, the width, of the space charge region is defined by $R-x$, where $R$ is the radius of the nanorod and $x$ is the distance of the edge of the SCR to the centre of the rod.

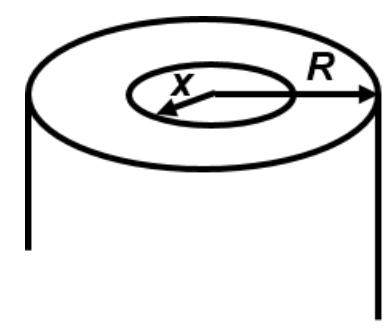

Figure 4. The space charge region in a partially depleted nanorod exists between the surface of the nanorod and a distance $x$ from the centre, where $x$ is related to the voltage drop across the space charge region by equation 10 .

In the abrupt depletion approximation, the space charge per unit surface area of the nanorod is given by

$$
Q_{S C R, \operatorname{rod}}=\frac{1}{2} q N_{d}\left(R^{2}-x^{2}\right)
$$

The electric field $E(r)$ in the radial direction across the SCR can be found by application of the Gauss theorem.

$$
E(r)=\frac{Q_{S C R, r o d}}{\varepsilon \varepsilon_{0}}=\frac{q N_{d}}{2 \varepsilon \varepsilon_{0}}\left(r-\frac{x^{2}}{R}\right)
$$

Integration between $r=x$ and $r=R$ then gives the potential drop across the space charge layer

$$
\Delta \phi_{S C R, \operatorname{rod}}=\frac{N_{d} q}{2 \varepsilon \varepsilon_{0}}\left[\frac{1}{2}\left(x^{2}-R^{2}\right)+x^{2} \ln \left(\frac{R}{x}\right)\right]
$$

This expression differs from equation 3 in Mora-Sero et al.[19], but is identical with equation 9.64 in Bisquert.[22]. The corresponding space charge capacitance is obtained by calculating the derivatives of the space charge and $\Delta \phi_{S C R \text {,rod }}$ with respect to $x$ to obtain the required derivative of the space charge with respect to the voltage drop.

$$
C_{S C, \text { rod }}=\frac{d Q_{S C R, \text { rod }}}{d \Delta \phi_{S C R, \text { rod }}}=\frac{d Q_{S C R, \text { rod }}}{d x} \frac{d x}{d \Delta \phi_{S C R, \text { rod }}}=\frac{\varepsilon \varepsilon_{0}}{\operatorname{Rln}\left(\frac{R}{x}\right)}
$$

Again this expression differs from equation given in Mora-Sero et al.,[19] but is identical with equation 9.68 in Bisquert.[22] 
The width of the space charge region in the cylindrical case (defined as $R-x$ ) can now be obtained as a function of the voltage drop across the space charge layer using equation 10 . Whereas equation 1 predicts that $W_{S C R}$ varies with the square root of band bending without limit, the variation in the cylindrical case diverges and then saturates as the space charge expands to fill the whole cylinder and $W_{S C R}$ reaches the limiting value $R$. Figure 5 contrasts the potential dependence of the width of the space charge regions for the two electrode geometries. For the nanorods, the $\Delta \phi_{S C R}$ reaches a limiting value $\Delta \phi_{d e p l}$ when $W_{S C R, \text { rod }}=R$ and the rods are completely depleted of majority carriers. For further increases in potential, inversion can occur, and the charge region will collapse as mobile holes are formed.

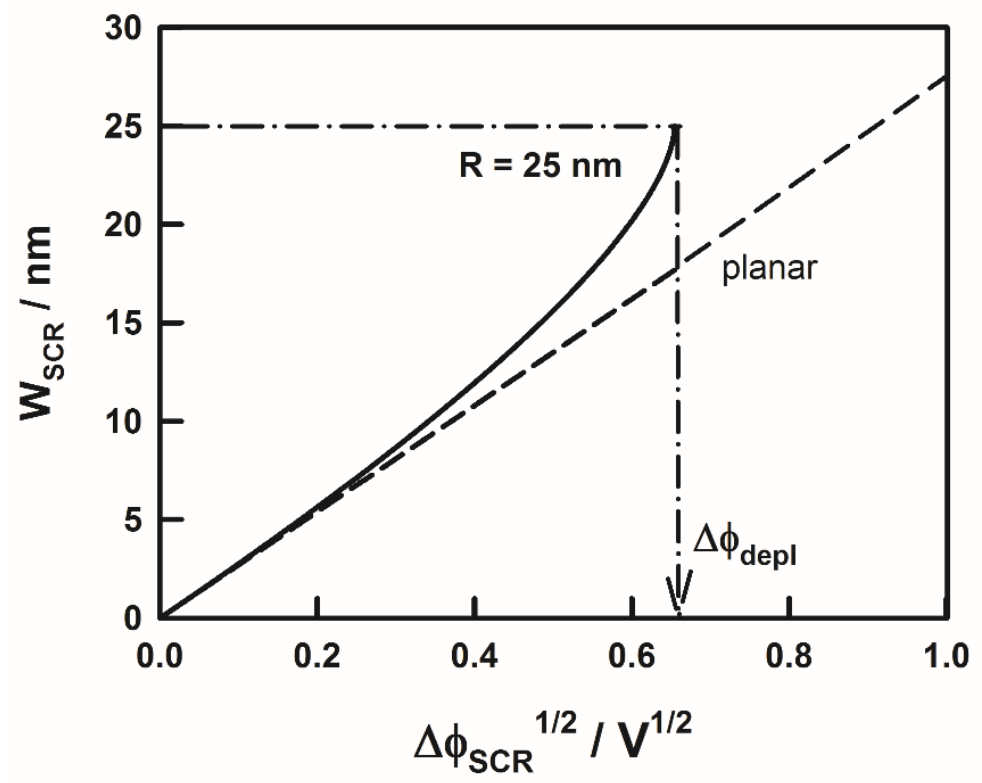

Figure 5. Comparison of the variation of space charge layer width for the planar and cylindrical cases. Note that the $x$-axis is the square root of the potential drop across the SCR. Nanorod radius $25 \mathrm{~nm}, N_{d}=5 \times 10^{18} \mathrm{~cm}^{-3}$, $\varepsilon_{\mathrm{Fe} 2 \mathrm{O} 3}=33$. In the case of the nanorod, full depletion is reached when the space charge region expands to occupy the entire volume $(\mathrm{x} \rightarrow 0)$.

Figure 6 illustrates the deviation from linear Mott Schottky behaviour predicted for nanorods with different radii. Even for rather high doping levels (here $10^{19} \mathrm{~cm}^{-3}$ ), the effects are severe when the nanorod radius is below $50 \mathrm{~nm}$, with the plot curving away from the tangent that corresponds to the Mott Schottky line for a planar electrode. 


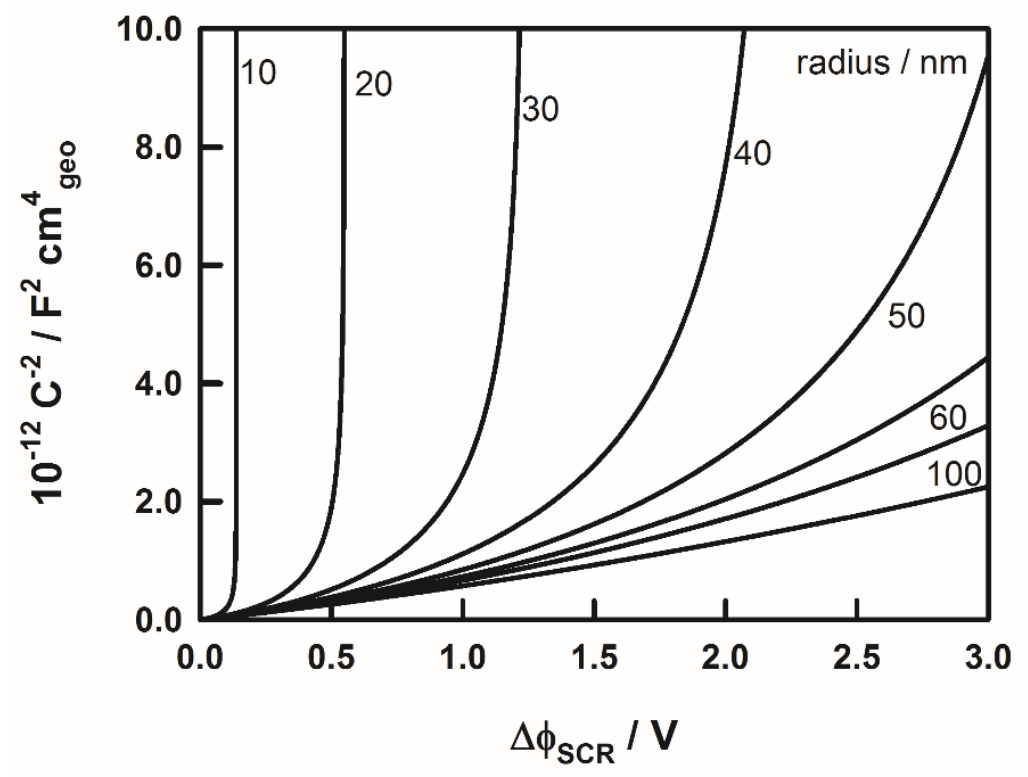

Figure 6. Mott Schottky plots of the space charge capacitance per unit area calculated as a function of the potential drop across the space charge region for nanorods of different radius. Values used in the calculation were $R(\mathrm{~nm})$ as shown, $\varepsilon=33, N_{d}=10^{19} \mathrm{~cm}^{-3}$. For this doping density, the rod radius needs to be considerably greater than $50 \mathrm{~nm}$ for the Mott Schottky plots to approach the planar limit given by equation 6 .

The transition towards full depletion $(x \rightarrow 0)$ is evident in these Mott Schottky plots as a steep upward curve as $C_{S C R}$ tends to zero approaching the limiting potential drop $\Delta \phi_{\text {depl }}$. Whether or not this transition to full depletion is seen in the experimental voltage window should depend on the doping density and rod radius. Figure 7 illustrates the dependence of $\Delta \phi_{d e p l}$ on $N_{d}$ for hematite nanorods with a radius of $25 \mathrm{~nm}$. Given that inversion becomes possible in in $\mathrm{Fe}_{2} \mathrm{O}_{3}$ when the band bending exceeds around $1 \mathrm{eV}$, we conclude that full depletion will not be observed experimentally for $25 \mathrm{~nm}$ radius nanorods if $N_{d}$ exceeds around $10^{19} \mathrm{~cm}^{-3}$.

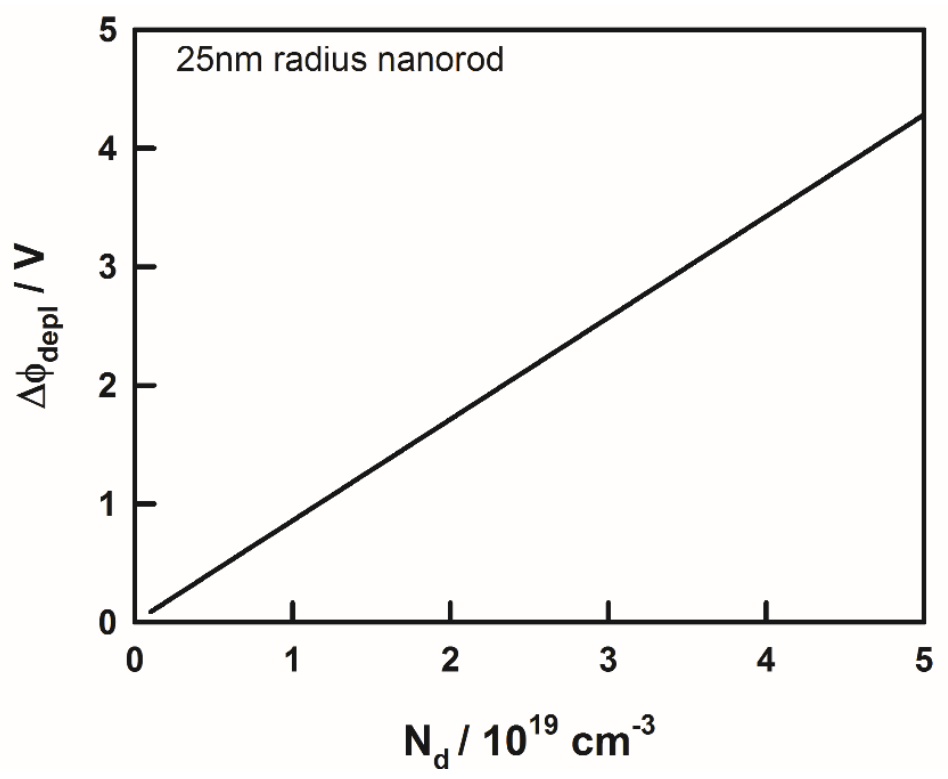

Figure 7. Dependence of the voltage $\Delta \phi_{\text {depl }}$ for full depletion on doping density calculated for hematite nanorods with radius $25 \mathrm{~nm}$. Note that if $\Delta \phi_{\text {depl }}$ is greater than around $1 \mathrm{~V}$, the transition to full depletion will not occur. Instead inversion is expected, giving rise to high dark currents. 
Figure 8 shows how $\Delta \phi_{\text {depl }}$ increases with rod radius for a given doping density $\left(10^{19} \mathrm{~cm}^{-3}\right)$. Following the same argument relating to inversion, for this doping density, experimental observation of full depletion should be limited to rods with radii less than $25 \mathrm{~nm}$.

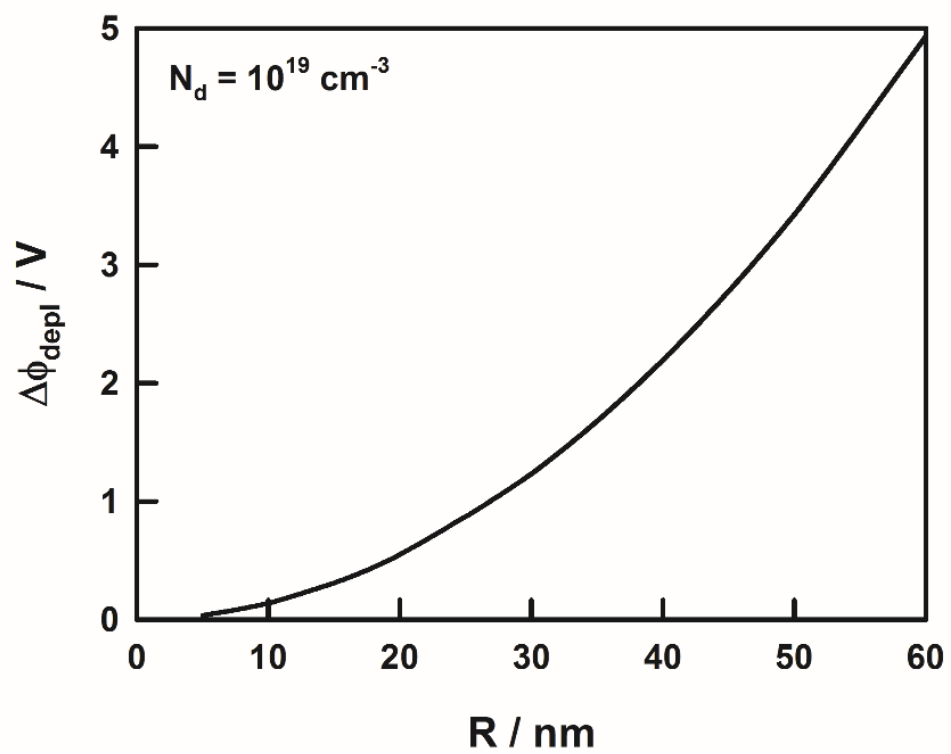

Figure 8. Dependence of $\Delta \phi_{\text {depl }}$ on rod radius $R$ calculated for hematite nanorods $N_{d}=10^{19} \mathrm{~cm}^{-3}$. Again, note that if $\Delta \phi_{\text {depl }}$ is greater than around $1 \mathrm{~V}$, the transition to full depletion will not occur. Instead inversion is expected, giving rise to dark currents as holes react with the electrolyte.

The high sensitivity of $\Delta \phi_{\text {depl }}$ to changes in rod radius shown by Figure 7 means that when there is a finite distribution of rod radii (as is obviously the case for the nanorods illustrated in Figure 2), the upwards transition in the Mott Schottky plot will not be as steep as shown in Figure 6. Smaller nanorods will deplete earlier than larger nanorods, leading to a more gradual increase in slope of the Mott Schottky plot with potential. If the distribution of radii is known, the corresponding Mott Schottky plot can be calculated.

We now turn to the overall potential distribution in the cylindrical system. As before, the total potential drop is divided between the potential difference across the SCR and across the Helmholtz layer (cf. equation 2). The potential drop across the Helmholtz layer is found by calculating the electric field at $r=R$ and applying the principle of dielectric continuity.

$$
\Delta \phi_{H, \text { rod }}=\frac{q N_{d}}{2 C_{H}}\left(\frac{R^{2}-x^{2}}{R}\right)
$$

where $C_{H}$ is the capacitance of the Helmholtz double layer. The total potential drop is the sum of the potential drops across the nanorod and across the Helmholtz layer. The derivation of equation 12 assumes that the permittivity (and hence the capacitance) of the Helmholtz region is independent of the electric field in the Helmholtz region. However, for high fields (> $4 \times 10^{6} \mathrm{~V} \mathrm{~cm}^{-1}$ [23]), dielectric saturation begins to reduce the dielectric constant of water. This effect is important for metal electrodes, where the field strengths exceed $10^{7} \mathrm{~V} \mathrm{~cm}{ }^{-1}$, leading to Helmholtz capacitances below $20 \mu \mathrm{F} \mathrm{cm} \mathrm{cm}^{-2}$ in the limit of full saturation. If we consider hematite nanorods with a radius of $25 \mathrm{~nm}$ and a doping density of $10^{20} \mathrm{~cm}^{-3}$, the electric field strength at the boundary to the electrolyte (i.e. at $r \rightarrow R$ ) calculated from equation 9 is of the order of $10^{6} \mathrm{~V} \mathrm{~cm}^{-1}$, which is below the field at which saturation effects begin to lower the bulk dielectric constant of water (ca. 80 at $20^{\circ} \mathrm{C}$ ). We therefore expect the 
Helmholtz capacitance to be of the order of $200 \mu \mathrm{F} \mathrm{cm}$ (based on a notional double layer thickness of $0.35 \mathrm{~nm}$ ). This value has therefore been used in the calculations discussed here.

In the limit $x \rightarrow 0, C_{s c, \text { rod }} \rightarrow 0$, and the nanorod becomes completely depleted and the space charge capacitance becomes zero (in fact, this limit is not adequately described by the abrupt depletion approximation,[21] but this is unimportant for the development of the present discussion). Under full depletion conditions, photogenerated electron-hole pairs will still be separated in the space charge region, with holes moving towards the interface and electrons towards the centre of the rods. The extraction of electrons at the base of the rod will then generate a gradient of electrochemical potential that controls the flow of electrons towards the substrate by drift/diffusion. This generation collection-problem is outside the scope of the present discussion, but if the radius of the nanorods is sufficiently small (tens of $\mathrm{nm}$ ), we may expect effective shielding by the electrolyte, so that electron collection in the direction normal to the substrate will be driven predominantly by the concentration gradient of majority carriers (i.e. by the diffusion of electrons in the case of hematite nanorods). In situ characterization of the conductivity of the nanorods under these conditions will be difficult if not impossible.

Quite apart from the fact that inversion may occur before complete depletion, the prediction that the capacitance tends to zero as $W_{S C R} \rightarrow R$ is physically implausible. There must always be a geometric capacitance to consider. It appears that the residual geometric capacitance in the full depletion limit has not been considered explicitly, but Bisquert et al.[24] have modelled the field distribution for a fully depleted cylindrical nanorod as part of a more general analysis of the electric potential distribution in nonporous semiconductor electrodes. These authors show that the electrical field originating in the substrate penetrates only a short distance of the order of $R$ into the nanorod. This means that under full depletion conditions, the counter charge in the electrolyte will be restricted to a narrow 'collar' at the nanorod base. We therefore expect that the limiting geometric capacitance will correspond to charging a surface area of the order of the basal area of the nanorod and a separation of the order of the rod radius as illustrated in Figure 9.

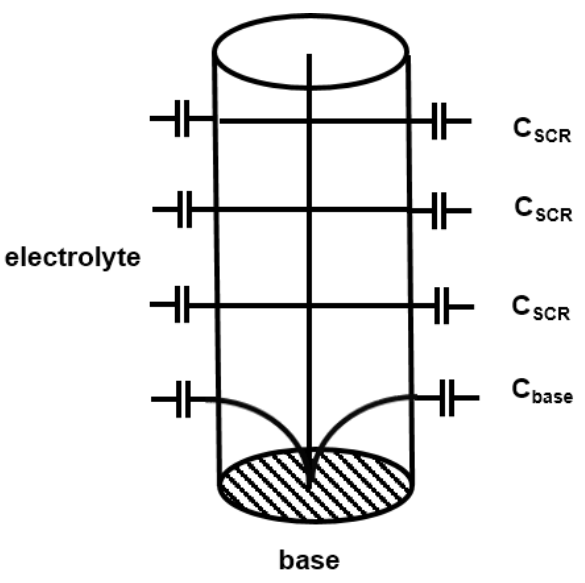

Figure 9. Schematic representation of the capacitance distribution in a cylindrical nanorod immersed in an electrolyte. The space charge capacitance $C_{S C R}$ is distributed along the cylinder and is connected between the central quasi neutral region and the electrolyte as shown. In addition, there is a geometric capacitance $C_{\text {base }}$ between the substrate at the base of the cylinder on one side and the electrolyte on the other. This capacitance remains when the space charge capacitance becomes small as the rod approaches the full depletion limit.

The geometric capacitance at the base of the nanorod will be of the order of 


$$
C_{\text {base }} \cong \frac{\pi R^{2} \varepsilon \varepsilon_{0}}{R}=\pi R \varepsilon \varepsilon_{0}
$$

This geometric capacitance should place a lower limit of the capacitance reached in the limit that the space charge region expands to fill the entire nanorod (i.e. for $x \rightarrow R$ ), replacing the limit that $x \rightarrow R, C_{s c \text {, rod }} \rightarrow 0$ implicit in equation 9 . For a hematite nanorod array with a radius of $25 \mathrm{~nm}$ and a number density of $3.5 \times 10^{10} \mathrm{~cm}^{-2}$, the total geometric capacitance of the rod bases will be of the order of $0.5 \mu \mathrm{F} \mathrm{cm} \mathrm{cm}^{-2}$. The corresponding Helmholtz capacitance of the 'collar' regions will be of the order of $\pi N_{\text {rod }} R^{2} C_{H} \approx 140 \mu \mathrm{F} \mathrm{cm}{ }^{-2}$ (geometric) for $C_{H}=200 \mu \mathrm{F}$ $\mathrm{cm}^{-2}$. Therefore, under conditions of full depletion, we can neglect the potential drop in the Helmholtz layer at the base of the nanorods.

For an array of identical nanorods of length $L$ with a number density of $N_{\text {rod }} \mathrm{cm}^{-2}$, the ratio of total internal surface area to geometric surface area is $2 \pi N_{\text {rod }} R L$. It follows that the rod space charge, rod base and rod Helmholtz capacitances referred to the geometric electrode area are respectively

$$
\begin{aligned}
& C_{s c, \text { geo }}=2 \pi N_{\text {rod }} L \frac{\varepsilon \varepsilon_{0}}{R \ln (R / x)} \\
& C_{\text {base }} \approx \pi N_{\text {rod }} \varepsilon \varepsilon_{0} R \\
& C_{H, \text { geo }}=2 \pi R N_{\text {rod }} L C_{H}
\end{aligned}
$$

To model the transition from the situation where the width of the space charge region is smaller than the rod radius to the fully depleted situation semi-quantitatively, we place the geometric capacitance in parallel with the series combination of the nanorod space charge and Helmholtz capacitances. However, we also need to recognize that the exposed substrate will also affect the measured capacitance, and this is considered in the next section.

\subsection{The effect of the exposed substrate area}

So far, we have not considered the area of the substrate that is not covered by the nanorods. Often this area will be covered by a thin precursor or seed layer of the nanorod material, but here we only deal with situation where the bare substrate is exposed. Let us assume that the nanorods are grown on a fluorine-doped tin oxide (FTO) layer on glass. For an array of identical nanorods with radius $R$ and number density $N_{\text {rod }}$, the factional uncovered area is given by

$$
\theta=1-N_{\text {rod }} \pi R^{2}
$$

For the hematite nanorod array discussed above, $N_{\text {rod }}=3.5 \times 10^{10} \mathrm{~cm}^{-3}$ and $R=25 \mathrm{~nm}$, giving $\theta=0.31$, slightly higher than the value for square packing $(0.22)$. The significant area of exposed FTO will clearly impact on the measured capacitance since we need to consider the parallel capacitance due to the FTO using equation 6 . The equivalent circuit used to model the total capacitance is therefore as shown in Figure 10. The capacitance of the lower parallel 'arm' of the circuit in Figure 10 can be calculated using equation 6 with appropriate input values of the doping density, flat band potential and Helmholtz capacitance for the FTO. We note in passing that for the values used in the present calculations for hematite, the effect of $C_{\text {base }}$ is quite small because the internal area of the nanorods is nearly two orders of magnitude higher than the exposed FTO area. 


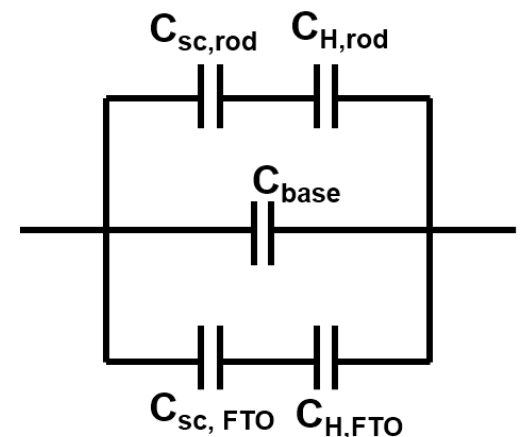

Figure 10. Dark equivalent circuit for a nanorod array on an FTO substrate. The circuit excludes resistive elements associated with charge transfer, i.e. it is assumed that the electrode potential range is restricted to values where no Faradaic reaction takes place (no inversion). In the case where a compact under-layer of the nanorod material is present (e.g. as a seed layer), an additional series capacitance is needed in the lowest arm of the circuit. See Figure 11 for typical values of the different capacitances.

Figure 11 illustrates typical magnitudes for the different capacitances in Figure 10. It is clear that the base capacitance and FTO capacitance are considerably smaller than the space charge capacitance until complete depletion occurs. At the same time, the Helmholtz capacitance is much larger than the space charge capacitance. This means that the measured capacitance should primarily reflect the space charge capacitance of the nanorod array.

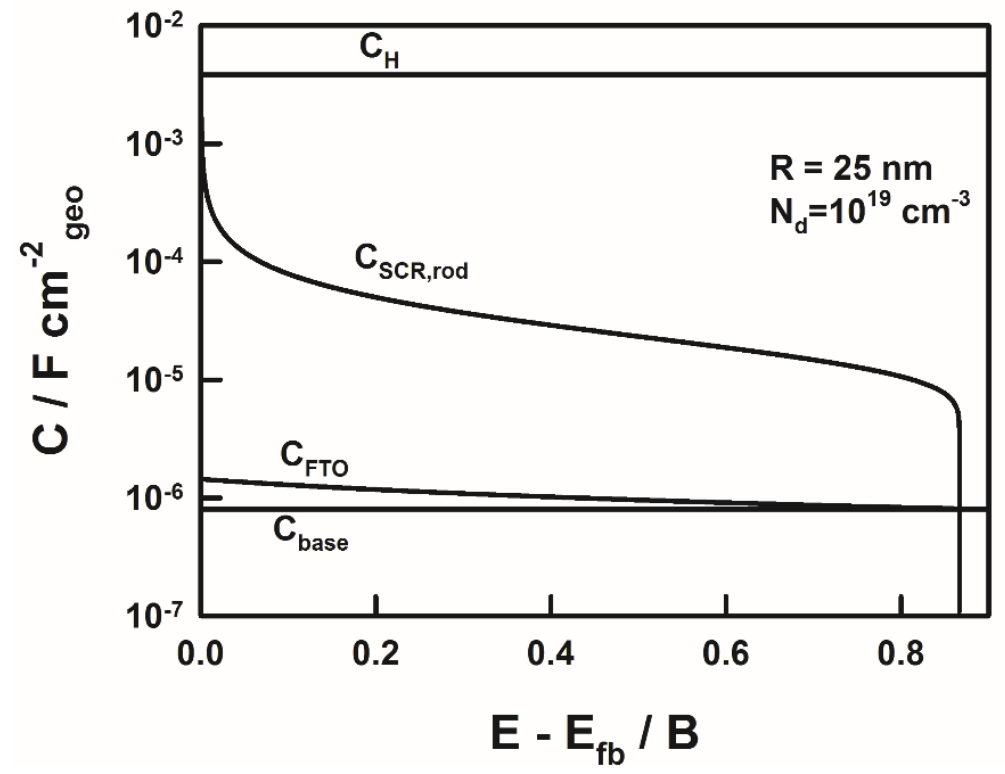

Figure 11. Voltage dependence of the different capacitances shown in Figure 10. $N_{\text {rod }}=3.5 \times 10^{10} \mathrm{~cm}^{-3}(\theta=$ $0.313), \varepsilon_{F e 2 O 3}=33, C_{H}=200 \mu \mathrm{F} \mathrm{cm}{ }^{-2}, N_{d}$ (nanorods; $\mathrm{cm}^{-3}$ ) as shown, $N_{d}(\mathrm{FTO})=10^{20} \mathrm{~cm}^{-3}, \varepsilon_{F T O}=12, E_{f b}(\mathrm{FTO})$ $E_{f b}\left(\mathrm{Fe}_{2} \mathrm{O}_{3}\right)=-0.4 \mathrm{~V}$. Capacitances expressed per unit of geometric electrode area.

Figure 12 illustrates the effect of the parallel FTO capacitance on the total measured capacitance of the system. As the cylinders become fully depleted, we expect the Mott Schottky plot to show a sharp upward transition to the Mott Schottky plot corresponding to exposed FTO. However, observation of this transition will again be limited by the onset of 
inversion as discussed above. In addition, such an abrupt transition requires that all the nanorods have the same radius. As pointed out above, a realistic distribution of rod radii will lead to significant broadening of the transition.

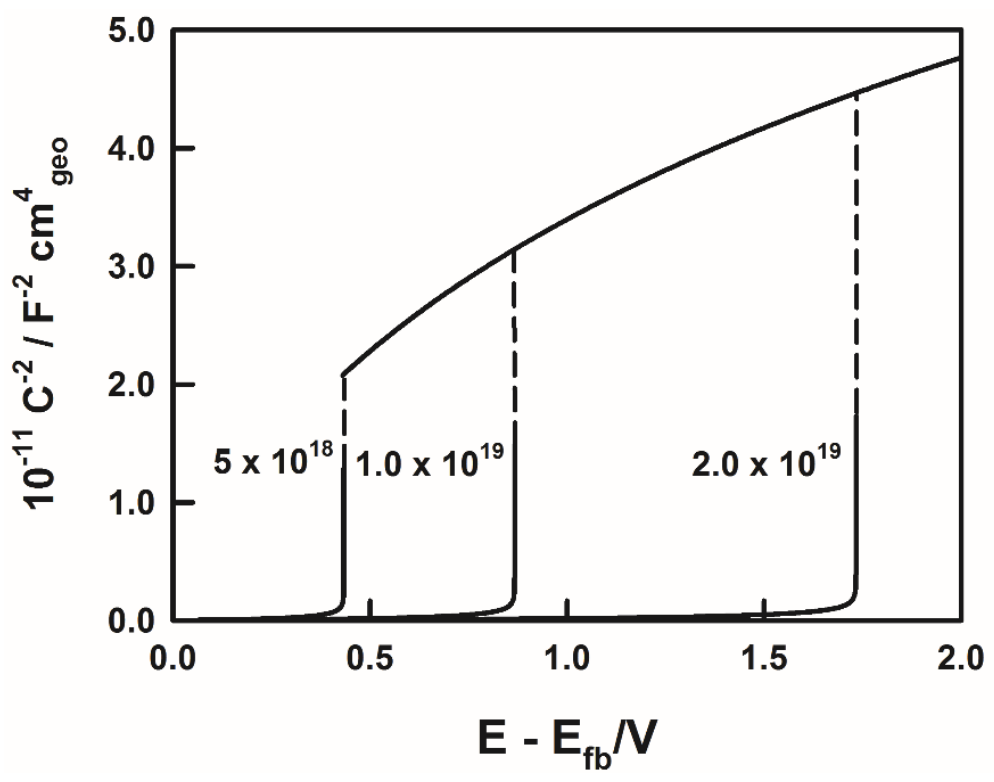

Figure 12. Mott Schottky plots calculated for an array of $25 \mathrm{~nm}$ radius hematite nanorods with a length of 350 $\mathrm{nm}$ illustrating the limit when the nanorods become fully depleted and the capacitance becomes determined mainly by the exposed FTO. Values as in Figure 11. Capacitances expressed per unit of geometric electrode area.

In the literature, it is not uncommon to encounter estimates of the doping density of nanorods or other nanostructured electrodes based on linear fits of Mott Schottky plots of the kind shown in Figure 3. In some cases, the internal surface area rather than the geometric surface area is considered, but in many cases even this factor is ignored. The analysis given here demonstrates that values of doping density obtained in these unsatisfactory ways are likely to be erroneous. Indeed, the complexity of the total capacitance plots makes fitting impossible unless a priori assumptions are made, or additional information is available. One approach is to measure the capacitance of the substrate without the nanostructured overlayer. If necessary, the sub-layer could include a seed and/or blocking layer if these are present. The fractional coverage $(1-\theta)$ of the substrate by the nanostructure can be estimated from SEM images. This is simplest if the nanostructure consists of identical elements such as nanorods or nanotubes. More complex structures such as those with 'cauliflower' morphologies[25] will be impossible to characterize quantitatively by impedance measurements unless 3-D numerical models are developed.

We now examine whether it is possible to fit the experimental capacitance data for the hematite nanorod array to obtain a reasonable estimate of the doping density. The first problem we encounter is that we no longer expect to be able to estimate the flat band potential reliably from the Mott Schottky plot. The previous discussion indicates that we do not obtain $E_{f b}$ from the linear fit shown in Figure 3. We could try taking a tangent to the curved initial part of the plot, but this will not give a reliable value either. Reported values of the flat band potential of hematite electrodes used for water splitting vary considerably - see, for example, the recent review by Hankin et al..[26] Given the problems of obtaining reliable Mott Schottky plots for with polycrystalline and nanostructured electrodes, we have turned to 
the work of Horowitz,[27] who carried out a very detailed impedance analysis of hematite crystals, paying particular attention to corrections needed to account for the potential drop in the Helmholtz layer in the case of highly doped electrodes. For $1 \mathrm{M} \mathrm{NaOH}$, Horowitz gives a flat band potential that corresponds to $0.124 \mathrm{~V}$ vs. RHE, and we have used this value in the fitting our experimental data for the hematite nanorod array.

Figure 13 illustrates how sensitive the fitting is to variation in the doping density. We have chosen to display the fit on a capacitance voltage plot rather than a Mott Schottky plot to avoid visual data compression and to aid comparison with Figure 1. The best fit is obtained for a doping density of $7.5 \times 10^{18} \mathrm{~cm}^{-3}$. The flat band potential value given by Horowitz was used in the calculation. The fit shown is insensitive to the capacitance of the FTO since the high internal surface area of the nanorods and the fact that inversion occurs before complete depletion ensure that the capacitance of the hematite is always much larger than that of the exposed FTO. However, the value of $N_{d}$ obtained should be treated with some caution since we have assumed, for example, that the internal surface area is equal to $2 \pi N_{\text {rod }} R L$, whereas some partial blocking of the gaps between the rods may occur. Uncertainties over the correct value of the relative permittivity of hematite will also affect the fitting, as will Fermi level pinning due to surface states.

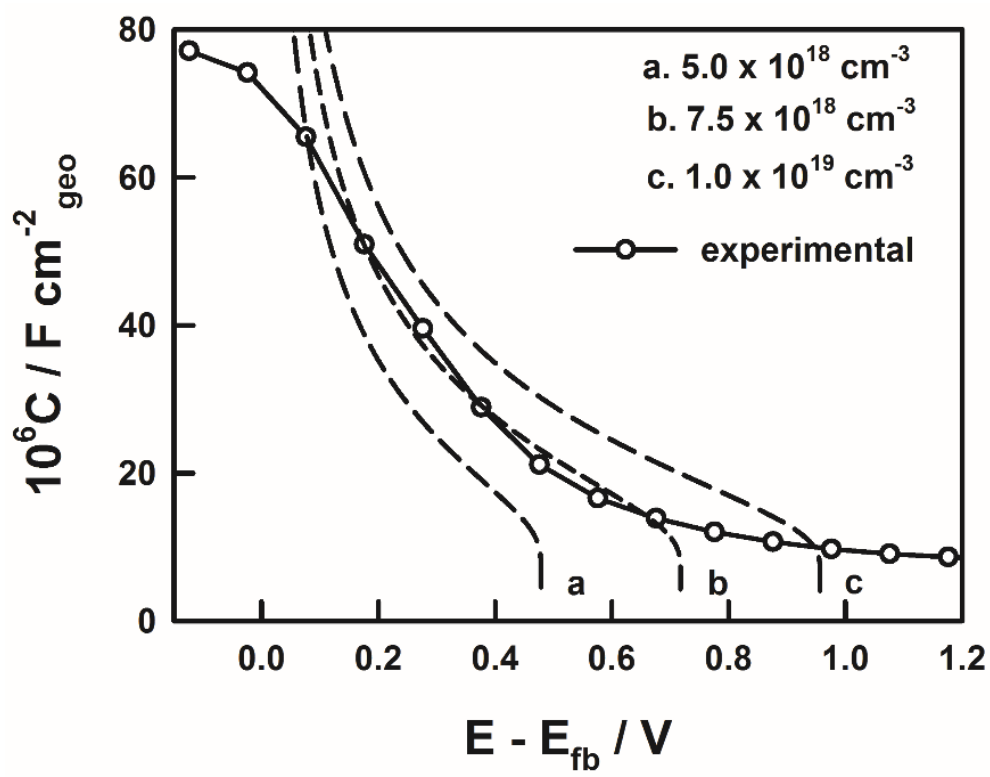

Figure 13. Comparison of experimentally measured capacitance of the hematite nanorod array (Figure 2) with calculations for three different values of $N_{d}$ as shown. Other values as in Figure 11.

The preceding analysis illustrates some of the difficulties inherent in attempts to derive reliable values of doping density and flat band potential from capacitance measurements on nanostructured electrodes. This is not to say that the measurement of capacitance has no value. Provided that we are dealing with a regular array rather than a random nanostructure and have sufficient additional information about dimensions and coverage, consideration of the appropriate space charge geometry can help us to obtain realistic values of $N_{d}$ and $E_{f b}$.

\section{How can nanostructuring enhance external photocurrent quantum efficiency?}

The external quantum efficiency (EQE) - also known as the IPCE (incident photon to current efficiency) - is a key measure of the performance of a photoelectrode. In the case of planar 
electrodes, the dependence of the EQE on incident photon energy and applied voltage is usually discussed in terms of the Gärtner equation.[13]

$$
E Q E=1-\frac{\exp \left[-\alpha(h v) W_{S C R}\right]}{1+\alpha L_{\min }}
$$

Here $\alpha(h v)$ is the absorption coefficient. The derivation of this equation assumes that all minority carriers arriving at the interface are transferred (i.e. take part in an electrochemical reaction in our case). Recombination in the SCR as well as at the surface is ignored. The contribution to the EQE made by minority carriers generated outside the SCR is reflected in the $1+\alpha L_{\min }$ term in equation 16. In the case of hematite, the term approaches 1 since the product $\alpha L_{\min }$ is much less than unity over the entire spectral range over which measurements are usually made (typically, $300-700 \mathrm{~nm}$ ). The problem we face when applying equation 16 to light-driven water splitting is that the rates of minority carrier reaction are slow since steps with high activation energies are involved in the multi-electron transfer processes leading to oxygen or hydrogen generation. Consequently, minority carriers accumulate in the space charge region and at the surface, where they can recombine with majority carriers. Electronhole recombination at the surface and in the space charge region therefore compete with charge transfer to the electrolyte, reducing the EQE. In order to apply equation 16 to the characterization of planar electrodes, it is necessary to use electron donor or acceptor species that react very quickly with minority carriers. In the case of n-type materials, $\mathrm{SO}_{3}{ }^{2-}$ ions or $\mathrm{H}_{2} \mathrm{O}_{2}$ can be used to scavenge holes sufficiently rapidly that no surface recombination occurs. Even if a scavenger is used, recombination in the SCR may still occur if minority carrier transport is slow due to low carrier mobility[28]. An alternative approach is to measure photocurrents using chopped or intensity-modulated light. If the frequency is high enough, recombination processes can be effectively 'frozen out'. The latter approach is the basis for intensity-modulated photocurrent spectroscopy (IMPS)[29, 30], which can be used to measure the rates of minority carrier reactions including charge transfer and recombination. IMPS has been used successfully to determine minority carrier lengths in III-V semiconductors using a simple analysis based on the Gärtner equation.[31]

This leads to the question whether we can apply the Gärtner approach to the case of nanostructured electrodes. We consider specifically the case of a nanorod array. Again, if the diffusion length of minority carriers is very small, then the EQE is simply determined by with the fraction of photons that are absorbed in the space charge region. It should be noted that this assumption requires that carriers are not lost by recombination in the SCR (see below). For a semi-infinite planar electrode with illumination from the electrolyte side the photon flux absorbed in the space charge region is simply the difference between the incident photon flux (corrected for reflection losses) and the flux of photons that exits the space charge region at $x=W_{S C R}$ so that $E Q E=1-e^{-\alpha W_{S C R}}$. For a nanorod array the situation is different since the space charge region extends in a radial direction throughout the cylinders, i.e. in a direction that is normal to the incident radiation. To obtain an idea of how the potential dependence of EQE will depend on the applied potential, we assume that the fraction of the incident light that is absorbed in the nanorod layer is given by $1-e^{-\alpha L}$, where $\mathrm{L}$ is the length of the nanorods. Light absorption may in fact be aided by multiple reflections, but we do not consider optical effects here. Furthermore, we make the same assumption as above, i.e. that the photocurrent is determined only by the fraction of the absorbed light incident light that is absorbed in the space charge region. For simplicity, we ignore the fraction of surface not 
covered by nanorods. The fraction of the total nanorod volume that is occupied by space charge is simply $1-\left(x^{2} / R^{2}\right)$, and hence the EQE is given by the product (cf. Figure 2 )

$$
E Q E=\left(1-e^{-\alpha L}\right)\left(1-\frac{x^{2}}{R^{2}}\right)
$$

where the dependence of $x / R$ on the potential drop in the space charge region is expressed by equation 10. Figure 14 contrasts the potential dependence of the EQE based on this simplified model with the behaviour predicted by the Gärtner equation for a planar electrode with the same doping density. The model predicts a large enhancement in all cases. Unfortunately, the steady state photocurrent voltage response of hematite electrodes under water splitting conditions is highly non-ideal as a consequence of surface recombination, so that a comparison of the predictions of the model with experiment will require the use of a suitable fast hole scavenger in order to bypass surface recombination.

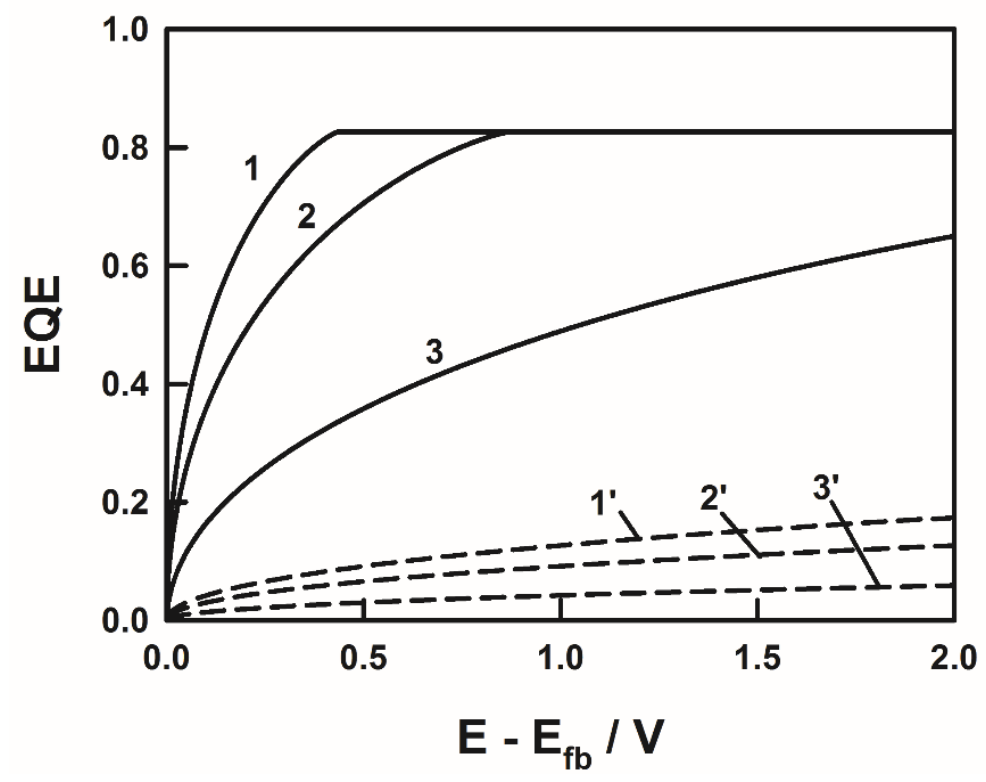

Figure 14. Comparison of the potential dependence of the EQE calculated for a nanorod array $(R=25 \mathrm{~nm}, L=$ $350 \mathrm{~nm}, \alpha=5 \times 10^{4} \mathrm{~cm}^{-1}$ ) using equation 17 with different values of $\left.N_{d}: 1\right) 5 \times 10^{18} \mathrm{~cm}^{-3}$, (2) $\left.10^{19} \mathrm{~cm}^{-3}, 3\right) 5 \times 10^{19}$ $\mathrm{cm}^{-3}$. The corresponding plots for planar electrodes calculated from the Gärtner equation (with $L_{\min }=0$ ) for the same doping densities are labelled 1', 2' and 3' respectively (broken lines). Note the substantial photocurrent enhancement brought about by changing the electrode geometry from planar to a nanorod array.

The enhancement brought about by nanostructuring should also be evident in EQE spectra. Figure 15 contrasts the predicted spectral responses of planar and nanorod electrodes. The spectra for the planar electrodes were calculated using the Gärtner equation taking into account the finite thickness of the layer (assumed to be the same as that of the nanorod array, i.e. $350 \mathrm{~nm}$ ). The calculation is based on the structured absorption coefficient spectrum reported by Marusak et al.[32] for highly oriented (110) hematite films. 


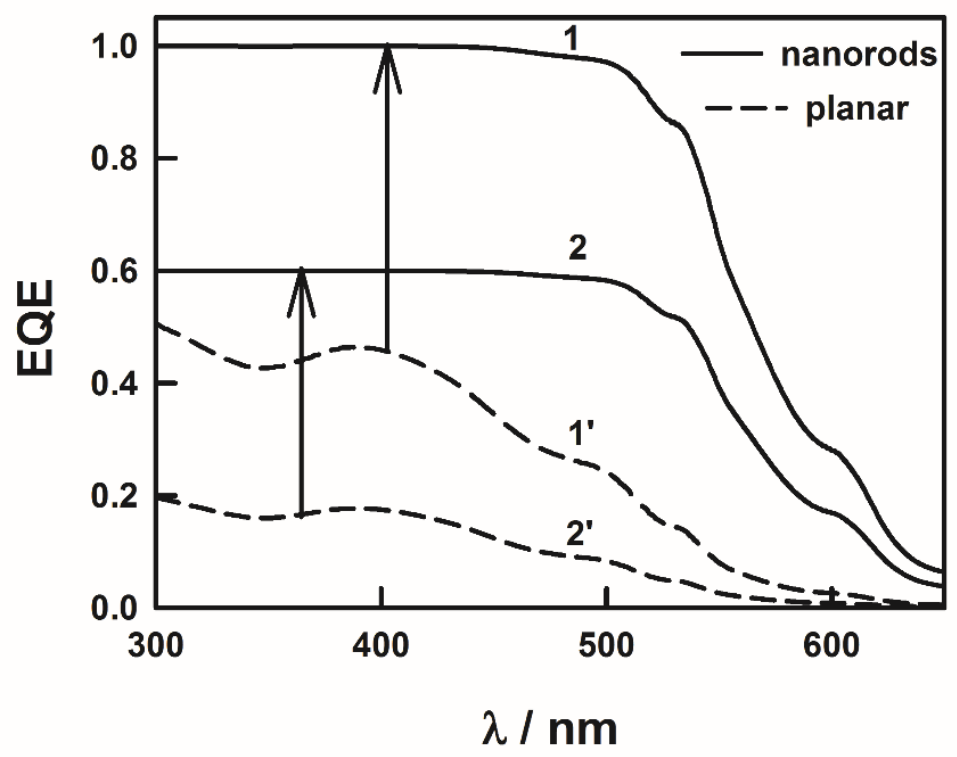

Figure 15. EQE spectra calculated for nanorod (full lines) and planar (broken lines) hematite electrodes of the same thickness $(350 \mathrm{~nm}) . R=25 \mathrm{~nm}$. Doping densities: 1) $\left.5 \times 10^{18} \mathrm{~cm}^{-3}, 2\right) 5 \times 10^{19} \mathrm{~cm}^{-3}$. Electrode potential 1.23 $\mathrm{V}$ vs. RHE. $E_{f b}$ (hematite) $=0.4 \mathrm{~V}$ vs. RHE. Note the strong EQE enhancement predicted in the case of the nanorod array. Absorption coefficient data taken from Marusak et al..[32] Note that it is assumed that all holes generated in the SCR are collected, even under full depletion conditions.

\section{Comparison of the EQE model with experiment in the case of hematite}

So how closely do experimental EQE spectra for nanostructured hematite electrodes resemble the calculated spectra in Figure 12? The answer is disappointing. Figure 16 shows that even the benchmark performance for nanostructured hematite electrodes reported by Kay al.[25] corresponds to an EQE spectrum that falls well below the ideal values. In the case of the three types of hematite nanorod electrodes studied by the present authors (undoped, Mn-doped and core-shell), the EQE response over most of the spectrum is also lower than predicted in the absence of surface and space charge recombination losses.

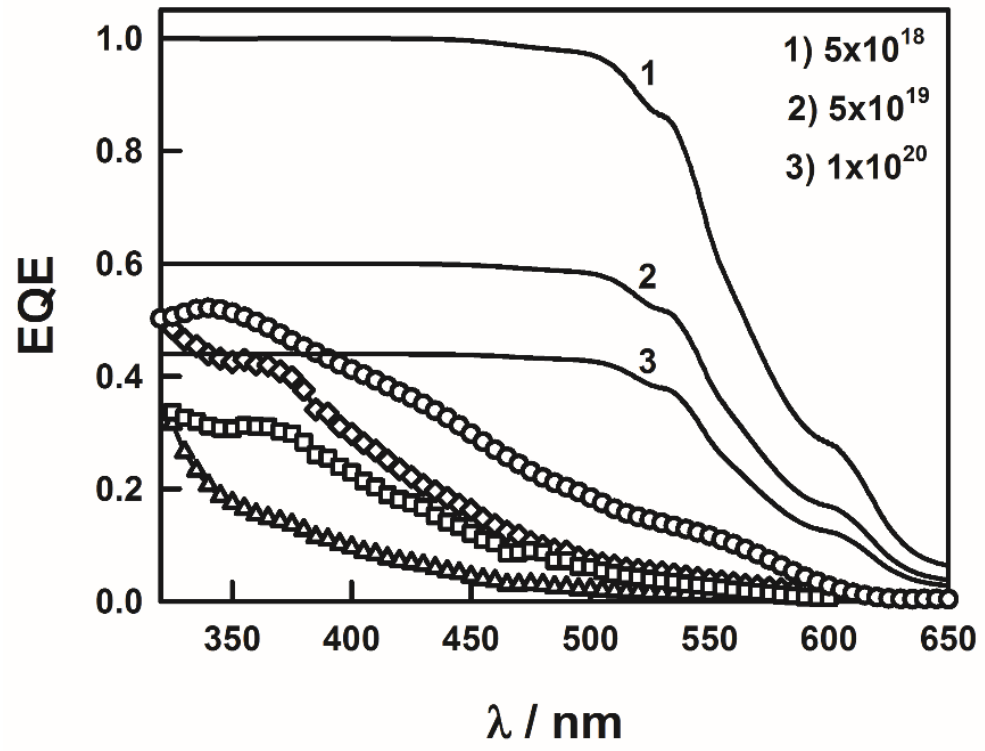

Figure 16. Comparison of experimental EQE spectra for different hematite films with the spectra calculated for $350 \mathrm{~nm}$ thick nanorod arrays with different doping densities (as shown $\mathrm{cm}^{-3}$ ). Circles: Kay et al.[25] 
'cauliflower' nanostructured hematite made by aerosol-assisted CVD; diamonds: Mn-doped hematite nanorods[33]; squares: core-shell hematite nanorods[16]; triangles: undoped hematite nanorods (this work).

This poor EQE response is reflected in the low short circuit current densities, $j_{s c}$, at $1.23 \mathrm{~V} \mathrm{Vs}$ RHE predicted by convoluting the EQE spectra with the AM1.5 G spectral photon flux distribution, As Table 1 shows, even the benchmark hematite electrode gives a $j_{s c}$ that is only around $25 \%$ of the value predicted for a hematite nanorod array $N_{d}=5 \times 10^{18} \mathrm{~cm}^{-3}$.

Table 1. Comparison of calculated and experimental values of short circuit current density at 1.23 V vs. RHE. The calculated $j_{s c}$ values were obtained by convoluting the EQE spectra for cylindrical geometry (obtained using the Gärtner approximation with $L_{\min }=0$ ) with the AM1.5G photon flux distribution.

\begin{tabular}{|l|l|}
\hline Electrode & $\mathbf{A M 1 . 5} \boldsymbol{j}_{\text {sc }} / \mathbf{m A ~} \mathbf{~ c m}^{-2}$ \\
\hline calculated, nanorods $N_{d} 5 \times 10^{18} \mathrm{~cm}^{-3}$ & 12.2 \\
\hline calculated, nanorods $N_{d} 5 \times 10^{19} \mathrm{~cm}^{-3}$ & 7.27 \\
\hline calculated, nanorods $N_{d} 1 \times 10^{20} \mathrm{~cm}^{-3}$ & 5.28 \\
\hline experimental, undoped hematite nanorods & 0.50 \\
\hline experimental, Mn-doped hematite nanorods & 1.56 \\
\hline experimental, core-shell hematite nanorods & 1.17 \\
\hline experimental, Kay et al.[25] nanostructured hematite & 2.78 \\
\hline
\end{tabular}

Since the experimental EQE spectra for the nanostructured hematite electrodes differ so substantially from those predicted using the simple model in which the active volume for charge separation is defined in terms of cylindrical geometry, we need to examine possible causes. Clearly a significant fraction of the holes photogenerated in the SCR is being lost by recombination, either at the surface or in the SCR itself. The competition between surface recombination and interfacial hole transfer at compact hematite electrodes interface has been studied by intensity-modulated photocurrent spectroscopy (IMPS)[34] and by photoelectrochemical impedance spectroscopy (PEIS). ${ }^{15}$ More recently, we have used IMPS to investigate three different types of hematite nanorods:[17] undoped, manganese-doped[16] and core-shell[33] hematite nanorods with a FeOOH coating The hole transfer efficiency $\eta_{\text {trans }}$, which represents the fraction of holes arriving at the surface that are successfully transferred, can be defined in terms of first order rate constants for charge transfer $\left(k_{\text {trans }}\right)$ and surface recombination $\left(k_{r e c}\right)$ as

$$
\eta_{\text {trans }}=\frac{k_{\text {trans }}}{k_{\text {trans }}+k_{\text {rec }}}
$$

Out study reveals that for all three types of nanorod electrode, $\eta_{\text {trans }}$ exceeds $95 \%$ at $1.23 \mathrm{~V}$ vs RHE. This is consistent with the observations that current voltage plots for hematite measured in water and the presence of hydrogen peroxide converge around this potential[35] since hydrogen peroxide is a fast hole scavenger (i.e. large $k_{\text {trans }}$ ). We can therefore exclude surface recombination as the explanation of the large difference between the calculated and experimental spectra.

A second possible explanation could be that surface states at the hematite/electrolyte interface lead to Fermi level pinning. These states can either be due to defects and impurities, or they could be intermediates or 'surface-trapped holes' generated by illumination. However, since the EQE is similar when a fast hole scavenger is used, the latter case seems unlikely to explain the losses. While partial Fermi level pinning certainly does occur at hematite electrodes, inspection of the calculated current voltage plots in Figure 14 suggests that high 
EQE values should be obtained for nanorod electrodes even if the band bending is much lower than expected from the measured flat band potential.

Whatever process is responsible for the loss of holes, it evidently involves a much shorter time scale than the range probed by IMPS (typically $10^{-6}-100 \mathrm{~s}$ ). Since the modelling already assumes that no holes are captured outside the SCR, we conclude that electron-hole recombination is likely to be occurring inside the SCR, even under conditions where holes are consumed rapidly by a hole scavenger like $\mathrm{H}_{2} \mathrm{O}_{2}$ when they arrive at the interface. The theory of IMPS for recombination in the SCR has been discussed by Vanmaekelbergh and deWit[36], but since it appears that surface recombination is normally dominant in semiconductor electrodes, the theory appears never to have been used to interpret IMPS data. These authors performed numerical calculations for a typical parameter values and found that the relaxation time constant was around 50 microseconds. Since this is shorter than the cell RC time constant[29], SCR recombination response will be submerged in the RC attenuation response and will therefore be difficult to identify. Recombination in the SCR has been discussed by Reichman[37] and by El Guibaly et al.[38] within the framework of the classical theory Sah, Noyce, and Shockley,[39] but a full discussion lies outside the scope of the present paper. In a recent comprehensive review of the status of water splitting at hematite electrodes, Zandi and Hamann[3] suggested that the transit time of holes through the SCR plays a key role in the performance of hematite photoanodes. These authors drew attention to neglected early work by Jarrett,[28] who showed that the Gärtner assumption that all minority carriers generated in the SCR reach the interface is no longer valid for semiconductors with low carrier mobility or very fast recombination. In the abrupt depletion approximation, the time taken for a minority carrier to move from the edge of the SCR to the surface is given by[28]

$$
\tau_{\text {transit }}=\frac{\varepsilon \varepsilon_{0}}{q N_{d} \mu}
$$

Jarrett makes the simplifying assumption that recombination throughout the SCR is first order so that it can be defined by a constant lifetime $\tau_{\text {recombination }}$. If $\tau_{\text {transit }}$ is less than $\tau_{\text {recombination }}$, the collection depth for holes will be less than the width of the space charge region. Very few experimental values of the hole mobility in hematite are available since it is difficult to dope it to become p-type. Hole transport in hematite by small polaron hopping has been modelled theoretically using Marcus theory to derive activation energies,[40] and the results suggest that hole mobilities are below $10^{-2} \mathrm{~cm}^{2} \mathrm{~V}^{-1} \mathrm{~s}^{-1}$. The corresponding transit times calculated from equation 18 for the undoped ${ }^{[17]}\left(N_{d}=5 \times 10^{18} \mathrm{~cm}^{-3}\right)$ and Mn-doped ${ }^{[17]}\left(N_{d}=1 \times 10^{20} \mathrm{~cm}^{-3}\right)$ hematite nanorods are therefore greater than 370 ps and 19 ps respectively. The reduction in transit time by a factor of 20 suggests that Mn-doping should lead to lower losses by recombination in the SCR (we find $N_{d}=10^{20} \mathrm{~cm}^{-3}$ for Mn-doped hematite). In the planar case, this beneficial effect will be partially offset by the fact that Mn doping reduces the width of the SCR by a factor of 4.5 (cf. equation 1), so that the enhancement achieved by nanostructuring becomes even more important. In fact, the EQE of the Mn-doped hematite nanorods is the highest of all three nanorod electrodes that we studied, which is consistent with this analysis.

A key parameter in the Jarret analysis is the hole collection depth, $L_{\text {coll }}$, which is determined by the relative values of $\tau_{\text {recombination }}$ and $\tau_{\text {transit }}$. If $L_{\text {coll }}<W_{S C}$, the EQE will be lower than the value predicted by the limiting form of the Gärtner equation (i.e. when $L_{\min } \rightarrow 0$ ). As Figure 15 
shows, the effect on the calculated EQE spectrum for the nanorods is simply to rescale them to lower values as $L_{\text {coll }}$ is reduced. A similar rescaling to lower values will also apply to the EQE spectra for planar electrodes (cf. Figure 12), i.e. the shape of the plots will be unaffected.

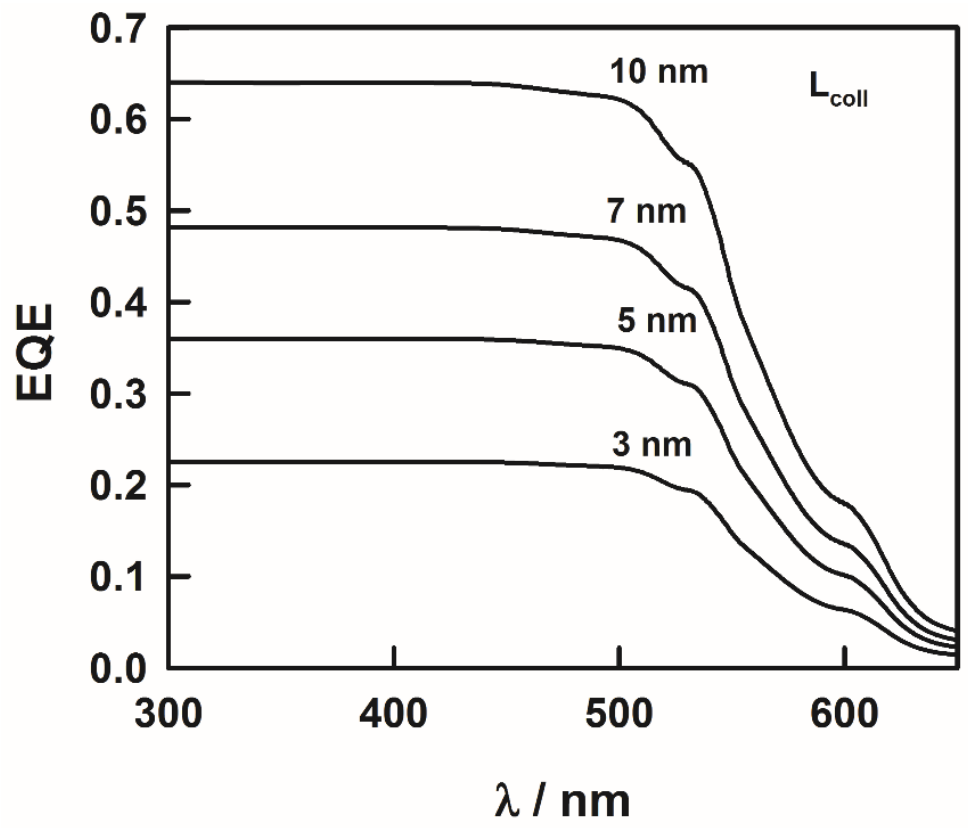

Figure 17. EQE spectra for $350 \mathrm{~nm}$ thick array of hematite nanorods $(R=25 \mathrm{~nm})$ calculated for different values of the hole collection depth showing the rescaling as $L_{\text {coll }}$ decreases.

The rescaling of EQE shown in Figure 15 does not provide an adequate explanation of why the shapes of the experimental EQE plots differ so much form those calculated for the cylinder model. The deviation could be due to the simplifying assumption that multiple internal reflections between the nanorods are ignored, but since the cauliflower structure of Kay et al.[25] has a similar shape, it seems more likely that another explanation is required. One intriguing possibility is that light absorption leads to a bound electron-hole pair and that the quantum efficiency for free carrier generation increases with the excess photon energy $h v$ - $E_{g}$. $[41,42]$ This would mean that the internal quantum efficiency depends on excess photon energy. To examine this hypothesis, in Figure 16 we have plotted the ratio of the experimental and predicted EQE values for the Mn-doped nanorod electrode as a function of photon energy. The analysis is necessarily approximate, since no account has been taken of reflection losses or of multiple internal reflections. Nevertheless, the plot suffices to show that the IQE increases linearly with photon energy between $2.5 \mathrm{eV}$ and $3.3 \mathrm{eV}$, before tending towards unity at higher photon energies. This behaviour is consistent with the suggestion that the creation of free electron hole pairs depends on the excess energy. 


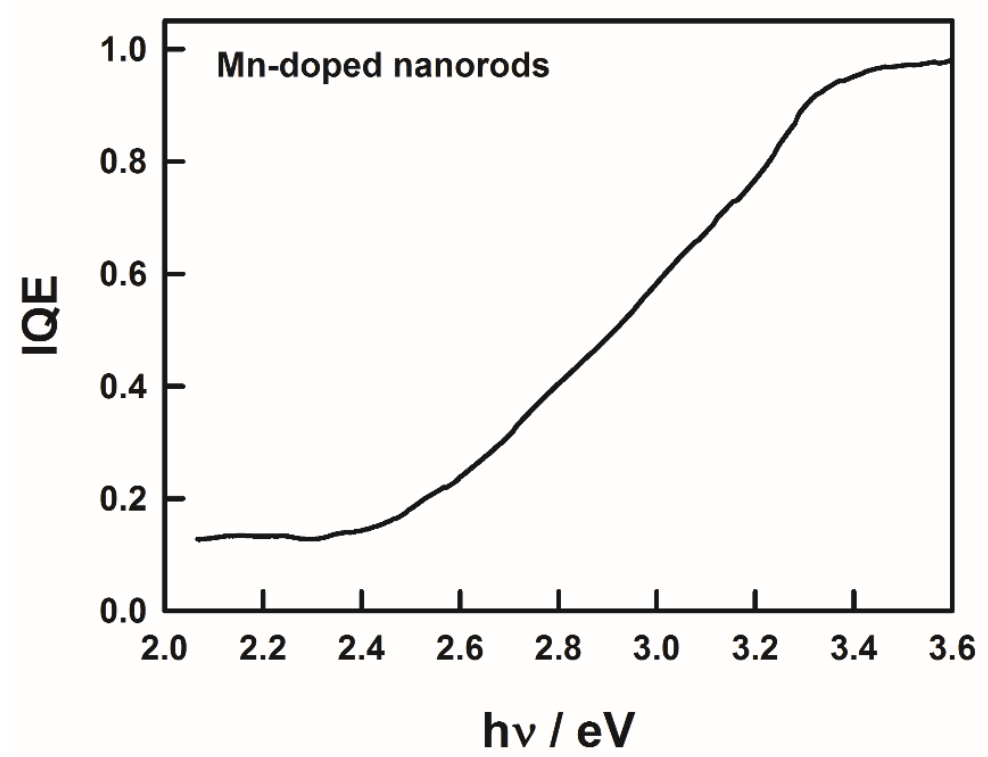

Figure 18. Dependence of the internal quantum efficiency (IQE) as a function of photon energy for Mn-doped nanorods. The IQE was calculated taking the ratio of the measured EQE to the EQE calculated using the simple model for cylindrical geometry (cf. equation 16).

To conclude this discussion, we note that oxide photoanodes with very short hole lifetimes and low hole mobilities are unlikely to achieve performances that are useful for light-driven water splitting. As we have shown elsewhere,[1] claims of 'high-efficiency' for nanostructured hematite electrodes frequently fail to stand up to close inspection. On a more positive note, a remarkable $\mathrm{EQE}$ of $90 \%$ with a square spectral response similar to the calculated plots for hematite shown in Figure 13 has been reported for 2.6 micron thick $\mathrm{WO}_{3}$ nanorod arrays $\left(R\right.$ ca. $140 \mathrm{~nm}$ ) coated with a $25 \mathrm{~nm}$ layer of $\mathrm{BiVO}_{4}$ and a cobalt catalyst.[43] The observed AM 1.5 photocurrent of $6.72 \mathrm{~mA} \mathrm{~cm}^{-2}$ at $1.23 \mathrm{~V}$ vs. RHE for this core-shell structure compares very favourably with the maximum expected for the wider bandgap $\mathrm{BiVO}_{4}(2.4 \mathrm{eV}$ as opposed to $2.1 \mathrm{eV}$ for hematite). This core-shell configuration, which corresponds to the extremely thin absorber (ETA) layer used in some types of solar cells, appears to offer a way of significantly enhancing the efficiency of hole collection in $\mathrm{BiVO}_{4}$, which also appears to suffer from slow hole transport.

Finally, we mention in passing the case of low-doped mesostructured n-type photoelectrodes where the feature size is so small (typically tens of $\mathrm{nm}$ ) that electron collection at the substrate becomes essentially diffusion-controlled because the electric field is small. The classical example is the mesoporous anatase electrode used in the dye-sensitized solar cell (DSC). When these electrodes are used for water splitting, there is evidence that electrons can be lost in transit to the substrate,[44] so that higher currents are obtained for substrate side illumination. The mechanism of 'recombination' in this case is not understood. It could involve, for example, surface-trapped holes or intermediates in the oxygen evolution reaction or electrons could be donated to dissolved oxygen.[45] Leng et al.[46] have treated this behaviour in terms of the electron diffusion length model developed for DSC, [47] where the electron acceptor is the tri-iodide ion, present in large excess in the electrolyte. However, it is unclear why the loss of electrons in a photoanode that is oxidizing water should follows the same pseudo-first order back reaction kinetics as in the DSC. So far, our attempts to model the loss of electrons in terms of electron-hole recombination considering the slow 
consumption of holes in the water splitting reaction have not been able to reproduce the observed first order behaviour.

\section{Conclusions}

This exploration of the properties of nanostructured electrodes illustrates the importance of critical examination of existing models and assumptions. Unfortunately, many papers dealing with nanostructured photoelectrodes focus on improved performance without asking questions about the mechanism of enhancement. The term' highly efficient' is often misused, and explanations of the enhancement mechanism are restricted to repetition of statements in the literature regarding matching feature size to the hole diffusion length. A simple analysis shows that simple geometric considerations alone predict EQE enhancement. However, in the case of hematite electrodes at least, the assumptions in the Gärtner model evidently do not apply. This may be because holes are lost by recombination during transit through the SCR to the interface. Alternatively, the internal quantum efficiency could be energy dependent as suggested above. Either way, the substantial enhancements expected for nanostructured electrodes are not fully realized. It is therefore difficult to escape the conclusion that unless new strategies to address these issues are developed, high efficiencies for water splitting will only be achieved with materials that have higher minority carrier mobilities and longer minority carrier lifetimes than oxides like hematite. It would therefore be interesting to test the relatively simple analytical model models developed here using well-defined silicon nanorods, for example.[48].

\section{Acknowledgments}

Financial support from the German Federal Ministry of Education and Research (BMBF project “CT-PEC", \#01DP14011) is gratefully acknowledged.

\section{References}

[1] L.M. Peter, Photoelectrochemical Water Splitting. A Status Assessment, Electroanalysis 27(4) (2015) 864-871.

[2] L.M. Peter, K.G.U. Wijayantha, Photoelectrochemical Water Splitting at Semiconductor Electrodes: Fundamental Problems and New Perspectives, Chemphyschem 15(10) (2014) 1983-1995.

[3] O. Zandi, T.W. Hamann, The Potential versus Current State of Water Splitting with Hematite, PCCP 17(35) (2015) 22485-22503.

[4] K.L.F. Sivula, F.; Grätzel, M., Solar Water Splitting: Progress using Hematite $\left(\alpha-\mathrm{Fe}_{2} \mathrm{O}_{3}\right)$ Photoelectrodes, ChemSusChem 4(4) (2011) 432-449.

[5] L.M. Peter, Energetics and Kinetics of Light-Driven Oxygen Evolution at Semiconductor Electrodes: the Example of Hematite, J. Solid State Electrochem. 17(2) (2013) 315-326.

[6] T.W. Hamann, Splitting Water with Rust: Hematite Photoelectrochemistry, Dalton Transactions 41(26) (2012) 7830-7834.

[7] F.W. Abdi, L. Han, A.H.M. Smets, M. Zeman, B. Dam, R. van de Krol, Efficient Water Splitting by Enhanced Charge Separation at a Bismuth Vanadate Tandem Electrode, Nature Communications 4 (2013) 3195. 
[8] Y. Liang, T. Tsubota, L.P.A. Mooij, R. van de Krol, Highly Improved Quantum Efficiencies for Thin Film BiVO 4 Photoanodes, J. Phys. Chem. C 115(35) (2011) 1759417598.

[9] F.F. Abdi, N. Firet, R. van de Krol, Efficient $\mathrm{BiVO}_{4}$ Thin Film Photoanodes Modified with Cobalt Phosphate Catalyst and W-doping, ChemCatChem 5(2) (2013) 490-496.

[10] J.H. Kennedy, K.W. Frese, Photooxidation of Water at $\alpha-\mathrm{Fe}_{2} \mathrm{O}_{3}$ Electrodes, J. Electrochem. Soc. 124(3) (1977) C130-C130.

[11] M.P. Dareedwards, J.B. Goodenough, A. Hamnett, P.R. Trevellick, Electrochemistry and Photoelectrochemistry of Iron(III) Oxide, J. Chem. Soc.-Faraday Trans. I 79 (1983) 2027-2041.

[12] H.C. Casey, B.I. Miller, E. Pinkas, Variation of Minority- Carrier Diffusion Length with Carrier Concentration in GaAs Liquid- Phase Epitaxial Layers, J. Appl. Phys. 44(3) (1973) 1281-1287.

[13] W.W. Gärtner, Depletion-Layer Photoeffects in Semiconductors, Physical Review 116 (1959) 84.

[14] R. De Gryse, W.P. Gomes, F. Cardon, J. Vennik, On the Interpretation of Mott- Schottky Plots Determined at Semiconductor/Electrolyte Systems, J. Electrochem. Soc. 122(5) (1975) 711-712.

[15] N. Sato, Electrochemistry at Metal and Semiconductor Electrodes, Elsevier, Amsterdam, 1998.

[16] Gurudayal, S.Y. Chiam, M.H. Kumar, P.S. Bassi, H.L. Seng, J. Barber, L.H. Wong, Improving the Efficiency of Hematite Nanorods for Photoelectrochemical Water Splitting by Doping with Manganese, ACS Appl. Mater. Interf. 6(8) (2014) 5852-5859.

[17] L.M.W. Gurudayal; Peter, Lydia H.; Abdi, Fatwa F., Revealing the Influence of Doping and Surface Treatment on the Surface Carrier Dynamics of Hematite Photoanodes, ACS Appl. Mater. Interf. (in press). (2017).

[18] R.A. Lunt, A.J. Jackson, A. Walsh, Dielectric Response of $\mathrm{Fe}_{2} \mathrm{O}_{3}$ Crystals and Thin Films, Chem. Phys. Lett. 586 (2013) 67-69.

[19] I. Mora-Sero, F. Fabregat-Santiago, B. Denier, J. Bisquert, R. Tena-Zaera, J. Elias, C. Levy-Clement, Determination of Carrier Density of $\mathrm{ZnO}$ Nanowires by Electrochemical Techniques, Appl. Phys. Lett. 89(20) (2006) 203117.

[20] R. Tena-Zaera, J. Elias, C. Lévy-Clément, C. Bekeny, T. Voss, I. Mora-Seró, J. Bisquert, Influence of the Potassium Chloride Concentration on the Physical Properties of Electrodeposited ZnO Nanowire Arrays, J. Phys. Chem. C 112(42) (2008) 16318-16323.

[21] J. Tornow, K. Ellmer, J. Szarko, K. Schwarzburg, Voltage Bias Dependency of the Space Charge Capacitance of Wet Chemically Grown ZnO Nanorods Employed in a DyeSensitized Photovoltaic Cell, Thin Solid Films 516(20) (2008) 7139-7143.

[22] J. Bisquert, Nanostructured Energy Devices. Equilibrium Concepts and Kinetics, CRC Press, Boca Raton, 2015, p 309.

[23] H.E. Alper, R.M. Levy, Field strength dependence of dielectric saturation in liquid water, J. Phys. Chem. 94(22) (1990) 8401-8403. 
[24] J. Bisquert, G. Garcia-Belmonte, F. Fabregat-Santiago, Modelling the Electric Potential Distribution in the Dark in Nanoporous Semiconductor Electrodes, J. Solid State Electrochem. 3(6) (1999) 337-347.

[25] A. Kay, I. Cesar, M. Grätzel, New Benchmark for Water Photooxidation by Nanostructured $\alpha-\mathrm{Fe}_{2} \mathrm{O}_{3}$ films, J. Am. Chem. Soc. 128(49) (2006) 15714-15721.

[26] A. Hankin, J.C. Alexander, G.H. Kelsall, Constraints to the Flat Band Potential of Hematite Photoelectrodes, PCCP 16(30) (2014) 16176-16186.

[27] G. Horowitz, Capacitance-Voltage Measurements and Flat-Band Potential Determination on $\mathrm{Zr}$-doped $\alpha-\mathrm{Fe}_{2} \mathrm{O}_{3}$ Single-Crystal Electrodes, J. Electroanal. Chem. Interfac. Electrochem. 159(2) (1983) 421-436.

[28] H.S. Jarrett, Photocurrent Conversion Efficiency in a Schottky Barrier, J. Appl. Phys. 52(7) (1981) 4681-4689.

[29] L.M. Peter, D. Vanmaekelbergh, Time and Frequency Resolved Studies of Photoelectrochemical Kinetics, in: R.C.K. Alkire, D.M. (Ed.), Adv. Electrochem. Sci. Eng., Weinheim, 1999, pp. 77-163.

[30] E.A. Ponomarev, L.M. Peter, A Generalized Theory of Intensity-Modulated Photocurrent Spectroscopy (Imps), J. Electroanal. Chem. 396(1-2) (1995) 219-226.

[31] R. Peat, L.M. Peter, Determination of the Electron-Diffusion Length in p-Gap By Intensity Modulated Photocurrent Measurements with an Electrolyte Contact, Appl. Phys. Lett. 51(5) (1987) 328-330.

[32] L.A. Marusak, R. Messier, W.B. White, Optical Absorption Spectrum of Hematite, $\alpha-$ $\mathrm{Fe}_{2} \mathrm{O}_{3}$ Near IR to UV, J. Phys. Chem. Solids 41(9) (1980) 981-984.

[33] Gurudayal, P.M. Chee, P.P. Boix, H. Ge, F. Yanan, J. Barber, L.H. Wong, Core-Shell Hematite Nanorods: A Simple Method To Improve the Charge Transfer in the Photoanode for Photoelectrochemical Water Splitting, ACS Appl. Mater. Interfac. 7(12) (2015) 68526859.

[34] L.M. Peter, K.G.U. Wijayantha, A.A. Tahir, Kinetics of light-driven oxygen evolution at alpha- $\mathrm{Fe}_{2} \mathrm{O}_{3}$ electrodes, Faraday Discuss. 155 (2012).

[35] O. Zandi, T.W. Hamann, Enhanced Water Splitting Efficiency Through Selective Surface State Removal, J. Phys. Chem. Lett. 5(9) (2014) 1522-1526.

[36] D. Vanmaekelbergh, A.R. Dewit, F. Cardon, Recombination in Semiconductor Electrodes - Investigation by the Electrical and Optoelectrical Impedance Method, J. Appl. Phys. 73(10) (1993) 5049-5057.

[37] J. Reichman, The Current-Voltage Characteristics of Semiconductor-Electrolyte Photovoltaic Cells, Appl. Phys. Lett. 36(7) (1980) 574-577.

[38] F. El Guibaly, K. Colbow, Theory of Photocurrent in Semiconductor-Electrolyte Junction Solar Cells, J. Appl. Phys. 53(3) (1982) 1737-1740.

[39] C.t. Sah, R.N. Noyce, W. Shockley, Carrier Generation and Recombination in P-N Junctions and P-N Junction Characteristics, Proc. IRE 45(9) (1957) 1228-1243.

[40] N. Iordanova, M. Dupuis, K.M. Rosso, Charge Transport in Metal Oxides: A Theoretical Study of Hematite $\alpha-\mathrm{Fe}_{2} \mathrm{O}_{3}$, J. Chem. Phys. 122(14) (2005) 144305-144305-10. 
[41] L.M. Carneiro, S.K. Cushing, C. Liu, Y. Su, P. Yang, A.P. Alivisatos, S.R. Leone, Excitation-Wavelength-Dependent Small Polaron Trapping of Photoexcited Carriers in $\alpha$ $\mathrm{Fe}_{2} \mathrm{O}_{3}$, Nat. Mater. 16(8) (2017) 819-825.

[42] D. Hayes, R.G. Hadt, J.D. Emery, A.A. Cordones, A.B.F. Martinson, M.L. Shelby, K.A. Fransted, P.D. Dahlberg, J. Hong, X. Zhang, Q. Kong, R.W. Schoenlein, L.X. Chen, Electronic and Nuclear Contributions to Time-Resolved Optical and X-ray Absorption Spectra of Hematite and Insights into Photoelectrochemical Performance, Energy Environment. Sci. 9(12) (2016) 3754-3769.

[43] Y.T. Pihosh, I.; Mawatari, K.; Uemura, J.;Kazoe, Y.;Koasr, S.;Makita, K.; Sugaya, T.; Matsui. T.; Fujita, D.; Tosa, M.; Kondo, M.; Takehiho, K., Photocatalytic Generation of Hydrogen by Core-Shell $\mathrm{WO}_{3} / \mathrm{BiVO}_{4}$ Nanorods with Ultimate Water Splitting Efficiency, Scientific Reports 5 (2015) 11141.

[44] H. Rensmo, H. Lindstrom, S. Sodergren, A.K. Willstedt, A. Solbrand, A. Hagfeldt, S.E. Lindquist, Photocurrent Losses in Nanocrystalline/Nanoporous $\mathrm{TiO}_{2}$ Electrodes due to Electrochemically Active Species in the Electrolyte, J. Electrochem. Soc. 143(10) (1996) 3173-3178.

[45] A. Hagfeldt, H. Lindstrom, S. Sodergren, S.E. Lindquist, Photoelectrochemical Studies of Colloidal Tio2 Films - the Effect of Oxygen Studied by Photocurrent Transients, J. Electroanal. Chem. 381(1-2) (1995) 39-46.

[46] W.H. Leng, P.R.F. Barnes, M. Juozapavicius, B.C. O'Regan, J.R. Durrant, Electron Diffusion Length in Mesoporous Nanocrystalline $\mathrm{TiO}_{2}$ Photoelectrodes during Water Oxidation, J. Phys. Chem. Lett. 1(6) (2010) 967-972.

[47] A. Hagfeldt, L.M. Peter, Characterization and Modelling of Dye-sensitized Solar Cells: A Toolbox Approach, in: K. Kalyanasundaranam (Ed.), Dye Sensitized Solar Cells, EPFL Press, Lausanne, 2010, pp. 457-554.

[48]. J.M. Foley, M.J. Price, J.I. Feldblyum, S. Maldonaldo, Analysis of the operation of thin nanowire electrodes for solar energy conversion. Energy Environ. Sci. 5 (2012) 5203-5220. 